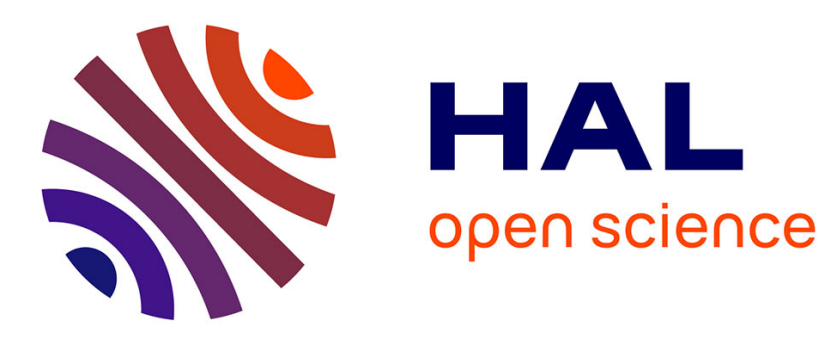

\title{
Gradient damage modeling of brittle fracture in an explicit dynamics context
}

Tianyi Li, Jean-Jacques Marigo, Daniel Guilbaud, Serguei Potapov

\section{To cite this version:}

Tianyi Li, Jean-Jacques Marigo, Daniel Guilbaud, Serguei Potapov. Gradient damage modeling of brittle fracture in an explicit dynamics context. International Journal for Numerical Methods in Engineering, 2016, 10.1002/nme.5262 . hal-01248263

\section{HAL Id: hal-01248263 \\ https://hal.science/hal-01248263}

Submitted on 29 Dec 2015

HAL is a multi-disciplinary open access archive for the deposit and dissemination of scientific research documents, whether they are published or not. The documents may come from teaching and research institutions in France or abroad, or from public or private research centers.
L'archive ouverte pluridisciplinaire HAL, est destinée au dépôt et à la diffusion de documents scientifiques de niveau recherche, publiés ou non, émanant des établissements d'enseignement et de recherche français ou étrangers, des laboratoires publics ou privés. 


\title{
Gradient damage modeling of brittle fracture in an explicit dynamics context
}

\author{
Tianyi $\mathrm{Li}^{1, *, \dagger}$, Jean-Jacques Marigo ${ }^{2}$, Daniel Guilbaud ${ }^{1,3}$ and Serguei Potapov ${ }^{1,4}$ \\ ${ }^{1}$ Institute of Mechanical Sciences and Industrial Applications, UMR EDF-CNRS-CEA-ENSTA ParisTech 9219 , \\ Université Paris Saclay, 828 boulevard des Maréchaux, 91762 Palaiseau Cedex, France \\ ${ }^{2}$ Laboratoire de Mécanique des Solides, École Polytechnique, 91128 Palaiseau Cedex, France \\ ${ }^{3}$ CEA Saclay, 91191 Gif-sur-Yvette Cedex, France \\ ${ }^{4}$ EDF Lab, 1 avenue du Général de Gaulle, 92141 Clamart Cedex, France
}

\begin{abstract}
SUMMARY
In this contribution we propose a large displacement extension of the dynamic gradient damage model. This last can be seen as a phase-field model for studying brutal fracture phenomena in quasi-brittle materials under impact-type loading conditions. Hencky logarithmic strain is adopted in the variational formulation to incorporate geometrical nonlinearities. The existing approaches to account for the tension-compression asymmetry of fracture behavior of materials are reviewed. A better understanding of these models is provided through a uniaxial traction experiment. We then give an efficient numerical implementation of the model in an explicit dynamics context. Simulations results obtained with parallel computing are discussed both from a computational and physical point of view. Different damage constitutive laws and tension-compression asymmetry formulations are compared with respect to their aptitude to approximate brittle fracture.
\end{abstract}

Received ...

KEY WORDS: gradient damage models; dynamic fracture; variational methods; explicit dynamics

\section{INTRODUCTION}

Phase-field modeling of dynamic fracture is gaining popularity over the last few years within the computational mechanics community [1, 2, 3, 4, 5]. From a physical point of view, it is based on the Variational Approach to Fracture as well as its elliptic regularization [6] which frees itself from several limitations inherent to the Griffith's theory: an initial crack and a given path. It settles down a unified framework covering the onset and the space-time propagation of cracks with possible complex topologies. A mere retranslation of Griffith's original idea, the variational formulation focuses on global energetic quantities and sees the crack evolution as a minimization movement of the sum of the stored elastic energy and the crack surface energy. Nucleation of defects in an initially sound domain as well as kinking, branching or coalescence of cracks naturally follow the competition of the involved energies. From a computational point of view, the introduction of a continuous phase field regularizes the sharp interface description of cracks, which renders unnecessary an explicit algorithmic tracking of the crack surface and the current crack front in 2-d and 3-d cases. Crack tip singularities automatically disappear due to regularization and the classical finite element

${ }^{*}$ Correspondence to: T. Li, IMSIA, UMR EDF-CNRS-CEA-ENSTA ParisTech 9219, Université Paris Saclay, 828 bd. des Maréchaux, 91762 Palaiseau Cedex, France.

†E-mail: tianyi.li@polytechnique.edu 
method can be used throughout the domain, as long as the regularized crack geometries are correctly captured by a relatively small mesh size. Due to these advantages, phase-field models can be used to explore numerous dynamic fracture phenomena in particular crack instabilities [7] or as a tool for experimental validations [8].

Meanwhile, the regularized phase-field formulation of fracture [6] can be acknowledged as a genuine gradient damage model per se, see [9,10,11], where the regularization parameter contributes to the fracture or damage behavior of materials. The link between the gradient damage model and the Griffith's theory of fracture without using global minimization arguments ( $\Gamma$-convergence theory for instance) is established in [12] for quasi-static situations. Its dynamic extension has been accomplished and will be the object of another contribution. Based on shape derivative techniques and an adaptation of the variational principles, a generalized Griffith's law is obtained which governs the crack tip equation of motion. When the material internal length is sufficiently small compared to the typical length of the structure, a separation of scales can be achieved in an asymptotic context. In that case, crack propagation in a gradient damage medium results from the competition between the energy release rate of the outer linear elastic fracture mechanics problem and the fracture toughness identified as the energy consumed during the damage band creation. Numerical verification of these theoretic ideas is conducted in [13] for an antiplane tearing experiment of a two-dimensional plate. The dynamic crack evolution obtained with the gradient damage model agrees well with the fracture mechanics predictions, both in absence or in presence of material inhomogeneities. The generalized energy release rates can be seen as a theoretical and numerical tool to establish the transition from damage to fracture.

In this contribution, we propose on one hand to formulate the dynamic gradient damage model under large displacement situations. Initially, these models are generally formulated under small displacement hypothesis (small strain and rotation), however for dynamic fracture problems finite strain theory should be preferred given the violence of impact-type loading conditions. Indeed, not only the material is experiencing strain localization inside the damage process zone, but also fast propagation of cracks of length comparable to that of the structure could result in the finite rotations of some fractured zones. On the other hand, our objective is to strengthen the bridge between the phase-field and the gradient-damage communities in order to achieve a better modeling of brittle dynamic fracture. We give a review of the existing approaches in both communities to account for the tension-compression asymmetry of fracture behavior of materials. A better understanding and comparison of these physical models is provided through analytical studies of a uniaxial traction experiment and their applications to real fracture problems. We also recognize the widely used regularized surface density function in the phase-field description of cracks as a special choice of the damage constitutive laws. The physical properties of these functions have been analytically studied in $[11,14,15]$. Here through concrete numerical simulations of dynamic fracture problems, we compare different damage constitutive laws with respect to their aptitude to approximate fracture, both from both the computational and physical point of view.

This paper is organized as follows. The variational formulation of the dynamic gradient damage model is recalled and extended to the large displacement case in Sect. 2. A review of the existing tension-compression asymmetry models is provided in Sect. 3, where they are compared against a uniaxial traction experiment. Sect. 4 is devoted to the spatial and temporal discretization of the model in an explicit dynamics context. We then discuss in Sect. 5 the simulation results which illustrate the differences of the previously presented damage constitutive laws and tension-compression asymmetry formulations. An experimental validation of the proposed model is also described. Finally some conclusions on the use of gradient damage models to approximate dynamic brittle fracture can be found in Sect. 6.

General notation conventions adopted in this paper are summarized as follows. Scalar-valued quantities will be denoted by italic roman or greek letters like the crack length $l_{t}$ or the damage field $\alpha_{t}$. Vector-valued (in $\mathbb{R}^{\mathrm{dim}}$ ) quantities will be represented by boldface letters such as the displacement field $\mathbf{u}_{t}$. Second or higher order tensors considered as linear operators will be indicated by sans-serif letters: the elasticity tensor $A$ for instance. Intrinsic notation is adopted and their contraction on lower-order tensors will be written without dots $A \boldsymbol{\varepsilon}=\mathrm{A}_{i j k l} \boldsymbol{\varepsilon}_{k l}$. Inner products between two vectors 
or tensors of the same order will be denoted with a dot, such as $A \boldsymbol{\varepsilon} \cdot \boldsymbol{\varepsilon}=\mathrm{A}_{i j k l} \boldsymbol{\varepsilon}_{k l} \boldsymbol{\varepsilon}_{i j}$. The spatial integration measures will be generally omitted since the usual Lebesgue or Hausdorff measures will be used for integration on the domain $\Omega$ or its boundary $\partial \Omega$. Time dependence will be noted at the subscripts of the involved quantities, as $\mathbf{u}:(t, \mathbf{x}) \mapsto \mathbf{u}_{t}(\mathbf{x})$. The symmetrized gradient operator is noted by $\nabla^{\mathrm{s}}=\frac{1}{2}\left(\nabla+\nabla^{\mathrm{T}}\right)$.

\section{VARIATIONAL FRAMEWORK AT LARGE DISPLACEMENTS}

The governing equations of the dynamic gradient damage model can be encapsulated into a purely variational framework thanks to the definition of a space-time action integral of several scalar energetic quantities of the structure, see [3,13]. The variational ingredients as well as the induced governing equations of the crack tip will be essentially the same to those of the linearized case. Cracks are located with the help of a scalar damage field $0 \leq \alpha \leq 1$ in a two or three-dimensional reference configuration $\Omega$, cf. Fig. 1. This field depicts a smooth transition between the undamaged part of the structure $\alpha=0$ and the crack $\alpha=1$, and thus can be considered as an isotropic phase-field indicator.
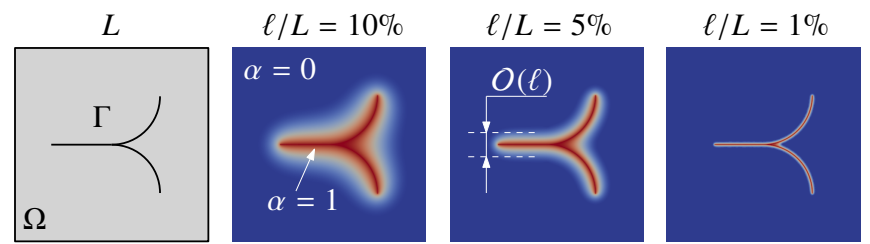

Figure 1. The discrete crack $\Gamma \subset \Omega$ approximated by a continuous damage field $0 \leq \alpha \leq 1$.

Given arbitrary admissible displacement, velocity and damage fields $\left(\mathbf{u}_{t}, \dot{\mathbf{u}}_{t}, \alpha_{t}\right)$ and a strain measure $\boldsymbol{\varepsilon}\left(\mathbf{u}_{t}\right)$ to be detailed later, we define respectively the elastic energy

$$
\mathcal{E}\left(\mathbf{u}_{t}, \alpha_{t}\right)=\int_{\Omega} \psi\left(\varepsilon\left(\mathbf{u}_{t}\right), \alpha_{t}\right)
$$

the kinetic energy

$$
\mathcal{K}\left(\dot{\mathbf{u}}_{t}\right)=\int_{\Omega} \frac{1}{2} \rho \dot{\mathbf{u}}_{t} \cdot \dot{\mathbf{u}}_{t}
$$

the external mechanical power of dead body forces $\mathbf{f}_{t}$ and dead surface tractions $\mathbf{F}_{t}$ applied on a subset of the boundary $\partial \Omega_{F}$

$$
\bar{W}_{t}\left(\mathbf{u}_{t}\right)=\int_{\Omega} \overline{\mathbf{f}}_{t} \cdot \mathbf{u}_{t}+\int_{\partial \Omega_{F}} \overline{\mathbf{F}}_{t} \cdot \mathbf{u}_{t}
$$

and the non-local (through the presence of the damage gradient) damage dissipation energy which will be related to the Griffith-like surface energy of cracks

$$
\mathcal{S}\left(\alpha_{t}\right)=\int_{\Omega} \varsigma\left(\alpha_{t}, \nabla \alpha_{t}\right)
$$

We observe that the elastic energy density $\psi$, the material density $\rho$ and the damage dissipation energy density $\varsigma$ are considered to be homogeneous in the reference configuration $\Omega$.

We have implicitly supposed a hyperelastic behavior for the underlying gradient damage material through the definition of a strain energy function in (1). Use of hypoelastic materials is also frequent in dynamic calculations due to their relatively low computational cost: only the stress increment $\Delta \sigma_{t}$ needs to be calculated given a strain increment $\Delta \boldsymbol{\varepsilon}_{t}$. However from a theoretic point of view, a good objective rate of the stress tensor should be carefully chosen for the hypoelastic law to be physically sound, which may complicates its numerical implementation [16]. Under quasi-static hypothesis 
authors of [17, 4] use a Lagrangian strain measure based on the right Cauchy-Green tensor $\mathbf{F}_{t}^{\top} \mathbf{F}_{t}$ for the finite-strain extension of phase-field models. It is a natural choice since the current configuration $\Omega_{t}$ is not known in advance for quasi-static calculations and the static equilibrium is written either in the initial reference configuration $\Omega=\Omega_{0}$ (total Lagrangian formulation) or in the last known reference configuration (updated Lagrangian formulation). In explicit dynamics however, dynamic momentum balance can be directly prescribed in the current configuration $\Omega_{t}$ which is calculated from the last iteration following the temporal discretization scheme. For this reason in this work we will use the Eulerian Hencky logarithmic strain tensor [18]

$$
\boldsymbol{\varepsilon}\left(\mathbf{u}_{t}\right)=\mathbf{h}_{t}=\log \mathbf{V}_{t}=\sum_{i}\left(\log \lambda_{i}\right) \mathbf{n}_{i} \otimes \mathbf{n}_{i}
$$

where $\mathbf{V}_{t}$ is the left stretch tensor from the polar decomposition $\mathbf{F}_{t}=\mathbb{I}+\nabla \mathbf{u}_{t}=\mathbf{V}_{t} \mathbf{R}_{t}$. Based on this strain measure, a simple Hookean type hyperelastic model [19] is adopted

$$
\begin{aligned}
& \psi_{0}\left(\mathbf{h}_{t}\right)=\frac{1}{2} \lambda\left(\operatorname{tr} \mathbf{h}_{t}\right)^{2}+\mu \mathbf{h}_{t} \cdot \mathbf{h}_{t}, \\
& \tau_{0}\left(\mathbf{h}_{t}\right)=\frac{\partial \psi_{0}}{\partial \mathbf{h}}\left(\mathbf{h}_{t}\right)=\lambda\left(\operatorname{tr} \mathbf{h}_{t}\right) \mathbb{I}+2 \mu \mathbf{h}_{t}
\end{aligned}
$$

where we emphasize that it is the Kirchhoff stress $\tau_{0}\left(\mathbf{h}_{t}\right)=J_{t} \boldsymbol{\sigma}_{0}\left(\mathbf{h}_{t}\right)$ with $J_{t}=\operatorname{det} \mathbf{F}_{t}$ the Jacobian determinant and not the Cauchy stress $\sigma_{0}$ that is derived from this strain energy $\psi_{0}$. It remains then to add the damage dependence into this elastic energy density. For formulational simplicities, we assume in this section that the damage acts isotropically on the undamaged elastic energy. Generalization to include tension-compression asymmetry (discussed in Sect. 3) should not influence the variational formulation proposed here. Hence, we arrive at the following elastic energy density

$$
\psi\left(\boldsymbol{\varepsilon}\left(\mathbf{u}_{t}\right), \alpha_{t}\right)=a\left(\alpha_{t}\right) \psi_{0}\left(\boldsymbol{\varepsilon}\left(\mathbf{u}_{t}\right)\right)=\frac{1}{2} a\left(\alpha_{t}\right) \mathrm{A} \boldsymbol{\varepsilon}\left(\mathbf{u}_{t}\right) \cdot \boldsymbol{\varepsilon}\left(\mathbf{u}_{t}\right)
$$

where $\mathrm{A}$ is the standard Hooke's elasticity tensor and $\alpha \mapsto a(\alpha)$ an adimensional damage constitutive function describing stiffness degradation in the bulk. This function should verify certain physical properties discussed in [11] for the gradient damage model to appropriately approximate brittle fracture. From (8), the stress tensor conjugate to the Hencky strain is thus the modulated Kirchhoff stress

$$
\boldsymbol{\tau}\left(\boldsymbol{\varepsilon}\left(\mathbf{u}_{t}\right), \alpha_{t}\right)=J_{t} \boldsymbol{\sigma}\left(\boldsymbol{\varepsilon}\left(\mathbf{u}_{t}\right), \alpha_{t}\right)=a\left(\alpha_{t}\right) \mathrm{A} \boldsymbol{\varepsilon}\left(\mathbf{u}_{t}\right) .
$$

As for the damage dissipation energy (4), we will use the same definition given in [11] (and a non-essential rescaling of the internal length $\ell \mapsto \sqrt{2} \ell$ ) since it measures the crack surface energy in the initial reference configuration

$$
\varsigma\left(\alpha_{t}, \nabla \alpha_{t}\right)=w\left(\alpha_{t}\right)+w_{1} \ell^{2} \nabla \alpha_{t} \cdot \nabla \alpha_{t} .
$$

In (10), the function $\alpha \mapsto w(\alpha)$ is another damage constitutive law representing the local energy dissipation during a homogeneous damage evolution and its maximal value $w(1)=w_{1}$ is the energy completely dissipated during such process. The internal length $\ell$, as can be seen in Fig. 1, controls the width of the damage band. It can be regarded as a purely numerical parameter which should be chosen as small as possible, see [6]. However its role as a material parameter is now fully recognized [20]. It is shown in $[11,14]$ that the critical stress or the maximal stress that can be attained by the material is proportional to the inverse square root of this parameter. A size effect is also introduced via this internal length where a one-dimensional bar of shorter length indeed turns out to be more stable. In [12], this internal length is recognized as a separator of scales between the damage process zone near the crack tip and the outer linear elastic fracture mechanics problem. An effective fracture toughness $G_{\mathrm{c}}$ can be identified as the energy dissipated during the optimal damage band creation

$$
G_{\mathrm{c}}=4 \ell \int_{0}^{1} \sqrt{w_{1} w(\beta)} \mathrm{d} \beta
$$


and (11) prescribes a relation between the fracture toughness $G_{\mathrm{c}}$, the energy dissipation due to damage $w_{1}$ and the internal length $\ell$.

Before announcing the governing equations of the gradient damage model, we need to specify the admissible spaces for the displacement and the damage evolutions in which the above energies (1)-(4) can be defined. We suppose that the admissible displacement space at time $t$ is an affine space of form $C_{t}=C_{0}+\mathbf{U}_{t}$ where $t \mapsto \mathbf{U}_{t}$ is a prescribed displacement on a subset $\partial \Omega_{U}$ of the boundary and $C_{0}$ is the associated vector space given by

$$
C_{0}=\left\{\mathbf{u}_{t}: \Omega \rightarrow \mathbb{R}^{\operatorname{dim}} \mid \mathbf{u}_{t}=\mathbf{0} \text { on } \partial \Omega_{U}\right\}
$$

in which the regularity condition is implicitly supposed. The admissible damage space will be built from an arbitrary damage state $0 \leq \alpha_{t} \leq 1$ and it is defined by

$$
\mathcal{D}\left(\alpha_{t}\right)=\left\{\beta_{t}: \Omega \rightarrow[0,1] \mid 0 \leq \alpha_{t} \leq \beta_{t} \leq 1\right\} .
$$

It can be seen that a virtual damage field $\beta_{t}$ is admissible, if and only if it is accessible from the current damage state $\alpha_{t}$ verifying the irreversibility condition. In order to use the Hamilton's principle for an arbitrary interval of time of interest $I=\left[t_{1}, t_{2}\right]$ we construct the following admissible evolution spaces

$$
\mathcal{C}(\mathbf{u})=\left\{\mathbf{v}: I \times \Omega \rightarrow \mathbb{R}^{\operatorname{dim}} \mid \mathbf{v}_{t} \in C_{t} \text { for all } t \in I \text { and } \mathbf{v}_{\partial I}=\mathbf{u}_{\partial I}\right\}
$$

and

$$
\mathcal{D}(\alpha)=\left\{\beta: I \times \Omega \rightarrow \mathbb{R} \mid \beta_{t} \in \mathcal{D}\left(\alpha_{t}\right) \text { for all } t \in I \text { and } \beta_{\partial I}=\alpha_{\partial I}\right\}
$$

by fixing the values of these two fields at both time ends denoted by $\mathbf{u}_{\partial I}=\left(\mathbf{u}_{t_{1}}, \mathbf{u}_{t_{2}}\right)$ and $\alpha_{\partial I}=\left(\alpha_{t_{1}}, \alpha_{t_{2}}\right)$. With all the variational ingredients set, we are now in a position to form the space-time action integral given by

$$
\mathcal{A}(\mathbf{u}, \alpha)=\int_{I} \mathcal{L}_{t}\left(\mathbf{u}_{t}, \dot{\mathbf{u}}_{t}, \alpha_{t}\right) \mathrm{d} t=\int_{I} \mathcal{E}\left(\mathbf{u}_{t}, \alpha_{t}\right)+\mathcal{S}\left(\alpha_{t}\right)-\mathcal{K}\left(\dot{\mathbf{u}}_{t}\right)-\overline{\mathcal{W}}_{t}\left(\mathbf{u}_{t}\right) \mathrm{d} t
$$

and annonce the following

Model 1 (Dynamic gradient damage evolution law)

The coupled two-field $(\mathbf{u}, \alpha)$ time-continuous dynamic gradient damage problem is governed by the following physical principles

1. Irreversibility: the damage $t \mapsto \alpha_{t}$ is a non-decreasing function of time.

2. First-order stability: the first-order action variation is non-negative with respect to arbitrary admissible displacement and damage evolutions

$$
\mathcal{A}^{\prime}(\mathbf{u}, \alpha)(\mathbf{v}-\mathbf{u}, \beta-\alpha) \geq 0 \text { for all } \mathbf{v} \in \mathcal{C}(\mathbf{u}) \text { and all } \beta \in \mathcal{D}(\alpha) .
$$

3. Energy balance: the only energy dissipation is due to damage

$$
\mathcal{H}_{t}=\mathcal{H}_{0}+\int_{0}^{t}\left(\int_{\Omega_{t}}\left(\sigma_{s} \cdot \nabla^{\mathrm{s}} \dot{\mathbf{U}}_{s}+\rho_{s} \ddot{\mathbf{u}}_{s} \cdot \dot{\mathbf{U}}_{s}\right)-\mathcal{W}_{s}\left(\dot{\mathbf{U}}_{s}\right)-\dot{\mathcal{W}}_{s}\left(\mathbf{u}_{s}\right)\right) \mathrm{d} s
$$

where the total energy is defined by

$$
\mathcal{H}_{t}=\mathcal{E}\left(\mathbf{u}_{t}, \alpha_{t}\right)+\mathcal{S}\left(\alpha_{t}\right)+\mathcal{K}\left(\dot{\mathbf{u}}_{t}\right)-\mathcal{W}_{t}\left(\mathbf{u}_{t}\right) .
$$

Further physical insights can be gained from the very compact first-order stability condition (16) written as a variational inequality. By developing the directional derivative of the action integral and supposing that the displacement field is sufficiently regular in time and in space, the classical elastodynamic equation can be derived (see Sect. A) for the Cauchy stress $\sigma_{t}=\boldsymbol{\sigma}\left(\boldsymbol{\varepsilon}\left(\mathbf{u}_{t}\right), \alpha_{t}\right)$ on the 
deformed configuration

$$
\begin{aligned}
& \rho_{t} \ddot{\mathbf{u}}_{t}=\operatorname{div} \sigma_{t}+\mathbf{f}_{t} \text { in } \Omega_{t}, \\
& \sigma_{t} \mathbf{n}=\mathbf{F}_{t} \text { on } \phi_{t}\left(\partial \Omega_{F}\right) .
\end{aligned}
$$

As for the governing equation for damage evolution, we observe that time dependence of damage is introduced solely via the irreversibility condition and the admissible damage evolution space $\mathcal{D}(\alpha)$. Using the fundamental lemma of calculus of variations and the fact that $\mathcal{D}\left(\alpha_{t}\right)$ defined in (12) is convex, the first-order stability tested with $\mathbf{v}=\mathbf{u}$ results in the minimality of the total energy corresponding to the current damage state $\alpha_{t}$ under the irreversible constraint, formally the same as for the quasi-static gradient damage model [11]

$$
\mathcal{E}\left(\mathbf{u}_{t}, \alpha_{t}\right)+\mathcal{S}\left(\alpha_{t}\right) \leq \mathcal{E}\left(\mathbf{u}_{t}, \beta_{t}\right)+\mathcal{S}\left(\beta_{t}\right) \text { for all } \beta_{t} \in \mathcal{D}\left(\alpha_{t}\right) .
$$

For the equivalent local conditions of the damage criterion (20) and the energy balance condition (17), readers are referred to [12]. Since the damage problem (20) is naturally defined in the (initial) reference configuration, the same local governing equations for damage will be derived compared to the small displacement case.

In this work we will consider two damage constitutive laws. Their use for phase-field modeling of cracks will be studied through numerical experiments in the sequel. They both involve only polynomial functions of the damage up to degree 2 , thus the elastic $\mathcal{E}$ as well as the damage dissipation energy $\mathcal{S}$ are quadratic with respect to damage, a rather interesting property from a computational cost point of view since the Hessian matrix is constant. Other sophisticated constitutive functions are proposed and analytically studied in $[15,14]$.

- Model with an elastic domain initially introduced in [11] and named after their initials. Damage does not evolve as long as a critical stress is not reached, a rather appreciated property when modeling brittle fracture. Then a stress-softening behavior is observed as damage grows, where the cubic and the quartic constitutive functions proposed by [21] still possess a small stress-hardening interval.

$$
a(\alpha)=(1-\alpha)^{2}, \quad w(\alpha)=w_{1} \alpha .
$$

- Original Ambrosio and Tortorelli regularization model introduced in [22]. The quadratic growing damage dissipation function $\alpha \mapsto w(\alpha)$ is used in many phase-field models of fracture $[2,1,3]$. This can be seen by calculating the maximal damage dissipation $w_{1}$ as a function of the fracture toughness $G_{\mathrm{c}}$ by (11). This constitutive function will be compared with (PAMM) through numerical simulations in Sect. 5.

$$
a(\alpha)=(1-\alpha)^{2}, \quad w(\alpha)=w_{1} \alpha^{2} .
$$

\section{TENSION-COMPRESSION ASYMMETRY}

In this section we will discuss several approaches in an attempt to account for the tension-compression asymmetry of damage behavior of materials. The objective is to provide a better understanding of the existing models following a theoretical approach and to point out some improvements that can be done in the future.

\subsection{Review of existing models}

In general two possibilities can be considered: modification of the elastic strain energy density (8), and/or modification of the variational principles (of irreversibility, stability and energy balance) outlined in Model 1. The second approach has been discussed in [23, 4] where the damage driving force $\partial_{\alpha} \psi\left(\varepsilon_{t}, \alpha_{t}\right)$ deduced from the energy minimization principle (20) is replaced by for example some stress-based criteria in presence of a damage threshold function. However it is known from [12] that the variational formulation plays an essential role in establishing the link between damage 
and fracture and in the definition of a generalized energy release rate with respect to the crack extension. That's why only the first possibility will be discussed in this exposition. Contrary to the small strain case, here the tension-compression asymmetry operates on the hyperelastic energy density (6) associated with the Hencky logarithmic strain $\boldsymbol{\varepsilon}\left(\mathbf{u}_{t}\right)$. By remarking that the trace of the Hencky strain (5) characterizes directly the local volume change at finite strains

$$
\operatorname{tr} \boldsymbol{\varepsilon}\left(\mathbf{u}_{t}\right)=\operatorname{tr} \log \mathbf{V}_{t}=\log \left(\lambda_{1} \lambda_{2} \lambda_{3}\right)=\log J_{t}
$$

where $\lambda_{i}$ are the principal stretches, we conclude with (6) that at $J_{t} \rightarrow 0^{+}$the Hencky elastic energy density goes to infinity, penalizing extreme compression. According to [17], automatically the material response with respect to damage should already be different under tension or compression.

From the literature survey of [24] on different phase-field like models for fracture and to the best knowledge of the authors, all existing approaches consist of partitioning the sound elastic energy $\psi_{0}(\boldsymbol{\varepsilon})$ in (6) into two part: a positive part $\psi_{0}^{+}(\boldsymbol{\varepsilon})$ which is considered to contribute to damage, and the negative part $\psi_{0}^{-}(\boldsymbol{\varepsilon})$ which resists to damage. We then replace the elastic energy density (8) symmetric in tension and compression by the expression

$$
\psi(\boldsymbol{\varepsilon}, \alpha)=a(\alpha) \psi_{0}^{+}(\boldsymbol{\varepsilon})+\psi_{0}^{-}(\boldsymbol{\varepsilon})
$$

where the damage degradation function $a(\alpha)$ only appears before the positive part $\psi_{0}^{+}(\boldsymbol{\varepsilon})$. By doing so, damage evolution is then driven by the positive elastic energy according to (20). We recall that the Kirchhoff stress $\tau\left(\varepsilon_{t}, \alpha_{t}\right)=J_{t} \sigma\left(\varepsilon_{t}, \alpha_{t}\right)$ is the thermodynamic variable associated to the Hencky logarithmic strain. It will be used as the main stress measure in the following analyses on tension-compression asymmetry formulations. Large strain effect (due to the presence of an inverted Jacobian determinant in front of the Cauchy stress) could be evaluated when particular material properties are known, however it should not influence the following qualitative results.

If furthermore the partition of the sound elastic energy $\psi_{0}(\varepsilon)$ is based on that of the strain tensor $\varepsilon=\varepsilon^{+}+\varepsilon^{-}$, i.e. the constitutive behaviors

$$
\begin{aligned}
& \boldsymbol{\varepsilon}^{ \pm} \mapsto \psi_{0}^{ \pm}\left(\boldsymbol{\varepsilon}^{ \pm}\right)=\frac{1}{2} \mathrm{~A} \boldsymbol{\varepsilon}^{ \pm} \cdot \boldsymbol{\varepsilon}^{ \pm}, \\
& \boldsymbol{\varepsilon}^{ \pm} \mapsto \tau_{0}^{ \pm}\left(\boldsymbol{\varepsilon}^{ \pm}\right)=\mathrm{A} \boldsymbol{\varepsilon}^{ \pm}
\end{aligned}
$$

are characterized by a same elasticity tensor $\mathrm{A}$ both for the positive and negative strains, then there exists in fact a local variational principle from which several existing tension-compression asymmetry models can be derived. This formulation is adapted from [10] where the framework of structured deformations is used to decompose the strain tensor into an elastic part and an inelastic one related to microstructures which in our notation is given by $\alpha \varepsilon^{+}$. However here we confine ourselves to macroscopic modeling and interpret the positive strain $\varepsilon^{+}$as the part that merely contributes to local material degradation. The mechanical modeling of such positive strains will be encapsulated into a convex subset $\mathcal{S}$ of the symmetrized 2nd-order tensor. The actual computation of $\boldsymbol{\varepsilon}^{+} \in \mathcal{S}$ is determined by the following local variational requirement for every material point

$$
\left\|\boldsymbol{\varepsilon}^{+}-\boldsymbol{\varepsilon}\right\|_{\mathrm{A}}=\min _{\mathbf{e} \in \mathcal{S}}\|\mathbf{e}-\boldsymbol{\varepsilon}\|_{\mathrm{A}}=\min _{\mathbf{e} \in \mathcal{S}} \mathrm{A}(\boldsymbol{\varepsilon}-\mathbf{e}) \cdot(\boldsymbol{\varepsilon}-\mathbf{e}) .
$$

Owing to the convexity of $\mathcal{S}$, the positive strain $\boldsymbol{\varepsilon}^{+}$is unique and is defined as the orthogonal projection of the total strain $\varepsilon$ onto the space $\mathcal{S}$ with respect to the energy norm defined by the elasticity tensor A. From convex analysis it is known that $\boldsymbol{\varepsilon}^{+}$that satisfies (24) can be equivalently characterized by

$$
-\mathrm{A}\left(\boldsymbol{\varepsilon}-\boldsymbol{\varepsilon}^{+}\right) \cdot\left(\mathbf{e}-\boldsymbol{\varepsilon}^{+}\right) \geq 0 \text { for all } \mathbf{e} \in \mathcal{S} .
$$

which implies from the definition (23) that the negative sound stress $\tau_{0}^{-}=\mathrm{A} \varepsilon^{-}$is in the polar cone $\mathcal{S}^{*}=\left\{\mathbf{e}^{*} \mid \mathbf{e}^{*} \cdot \mathbf{e} \leq 0\right.$ for all $\left.\mathbf{e} \in S\right\}$. If the space $\mathcal{S}$ is also a cone, i.e. closed with respect to arbitrary positive rescaling $\alpha \mathbf{e}$ for $\alpha>0$, then testing (25) with $\mathbf{e}=2 \boldsymbol{\varepsilon}^{+}$and $\mathbf{e}=\frac{1}{2} \boldsymbol{\varepsilon}^{+}$furnishes along with the symmetry of $A$ the following orthogonality conditions

$$
\begin{aligned}
& \tau_{0}^{-} \cdot \boldsymbol{\varepsilon}^{+}=\mathrm{A}\left(\boldsymbol{\varepsilon}-\boldsymbol{\varepsilon}^{+}\right) \cdot \boldsymbol{\varepsilon}^{+}=0 \\
& \tau_{0}^{+} \cdot \boldsymbol{\varepsilon}^{-}=\mathrm{A}\left(\boldsymbol{\varepsilon}-\boldsymbol{\varepsilon}^{-}\right) \cdot \boldsymbol{\varepsilon}^{-}=0 .
\end{aligned}
$$


Using (22) and (23), this implies that the total strain energy can be written by

$$
2 \psi(\varepsilon, \alpha)=a(\alpha) \tau_{0}^{+} \cdot \boldsymbol{\varepsilon}^{+}+\tau_{0}^{-} \cdot \boldsymbol{\varepsilon}^{-}
$$

where the crossed terms disappear thanks to (26). This provides another interpretation of (24) from a mechanical point of view: the positive part of the strain minimizes the negative part of the elastic energy $\boldsymbol{\tau}_{0}^{-} \cdot \boldsymbol{\varepsilon}^{-}$that resists to damage.

We now turn to the stress tensor derived from (22) and (23). In general we should have by definition

$$
\boldsymbol{\sigma}(\boldsymbol{\varepsilon}, \alpha) \mathbf{e}=a(\alpha) \tau_{0}^{+} \cdot \frac{\partial \boldsymbol{\varepsilon}^{+}}{\partial \boldsymbol{\varepsilon}}(\boldsymbol{\varepsilon}) \mathbf{e}+\tau_{0}^{-} \cdot \frac{\partial \boldsymbol{\varepsilon}^{-}}{\partial \boldsymbol{\varepsilon}}(\boldsymbol{\varepsilon}) \mathbf{e}
$$

where derivatives of the decomposed strains $\boldsymbol{\varepsilon}^{ \pm}$with respect to the total strain appear. Fortunately, as $\partial_{\varepsilon} \varepsilon^{+} \in \mathcal{S}$ and $\partial_{\varepsilon} A \varepsilon^{-} \in \mathcal{S}^{*}$, we have due to (25)

$$
\tau_{0}^{-} \cdot \frac{\partial \boldsymbol{\varepsilon}^{+}}{\partial \boldsymbol{\varepsilon}}(\boldsymbol{\varepsilon}) \mathbf{e} \leq 0 \text { and } \tau_{0}^{+} \cdot \frac{\partial \boldsymbol{\varepsilon}^{-}}{\partial \boldsymbol{\varepsilon}}(\boldsymbol{\varepsilon}) \mathbf{e} \leq 0
$$

By differentiating the orthogonality condition (26) with respect to the total strain $\varepsilon$, we find that the sum of the above two non-positive inner products equals to zero, which implies individually that these two expressions in (28) vanish. Recalling $\varepsilon=\boldsymbol{\varepsilon}^{+}+\boldsymbol{\varepsilon}^{-}$, (27) reads now

$$
\tau(\boldsymbol{\varepsilon}, \alpha) \mathbf{e}=\left(a(\alpha) \tau_{0}^{+}+\tau_{0}^{-}\right) \cdot \mathbf{e}
$$

from which the stress tensor is readily identified

$$
\tau(\varepsilon, \alpha)=a(\alpha) \tau_{0}^{+}+\tau_{0}^{-} .
$$

It can be noted that this expression is reduced to its negative part $\tau(\varepsilon, 1)=\tau_{0}^{-} \in \mathcal{S}^{*}$ for a totally damaged element.

Using this variational formulation (24), the modeling of material tension-compression asymmetry is thus reduced to the setting of such convex cone $\mathcal{S}$ destined to represent the strains that contribute to damage. Several existing phase-field like models of fracture can be derived within this framework [10].

- The original symmetric model of [22] can be trivially obtained by choosing $\mathcal{S}$ to all symmetric 2nd-order tensors. From (25) it can be deduced that $\boldsymbol{\varepsilon}^{+}=\boldsymbol{\varepsilon}$, i.e. the total strain contributes to damage irrespective of whether it corresponds to traction or compression.

- The deviatoric model of [9] is retrieved when $\mathcal{S}$ represents all symmetric 2nd-order tensors that have a zero trace (and the condition that $A$ is isotropic). Only deviatoric part of the strain $\operatorname{dev} \boldsymbol{\varepsilon}$ participates to damage. The negative stress $\tau_{0}^{-}$belongs to the polar cone of $\mathcal{S}$ which is characterized by a zero deviatoric part. Thus for a totally damaged element the stress is hydrostatic and has the form $p \llbracket$ for $p \in \mathbb{R}$.

- The model of [25] combines the previous two models by setting $\mathcal{S}$ to the first or the second one according to whether the trace of the total strain is positive tr $\varepsilon \geq 0$ or negative. Contrary to the linearized case, here thanks to (21) tr $\varepsilon \geq 0$ if and only if $J \geq 1$, i.e. corresponding to local volume expansion.

- The masonry-like model of [10] is obtained when $\mathcal{S}$ is chosen to include all positive semidefinite symmetric tensors. As $\mathcal{S}$ is a convex cone, the stress tensor can be simplified to (30) and hence the stress that can be attained by a totally damaged element is necessarily negative semidefinite, corresponding in fact to materials that do not support tension [17]. However the model as suggested by [10] with $\mathcal{S}$ containing all symmetric tensors of which all eigenvalues are greater than -1 may present some difficulties, as the orthogonality condition (26) and the simplified stress expression (30) no longer apply, $\mathcal{S}$ not being closed with respect to arbitrary positive rescaling. 
It can be noted that the widely used tension-compression asymmetry model of [26] adopts the elastic energy density split (22) but does not fit into the variational formalism (24). Denoting $\boldsymbol{\varepsilon}^{+}$ (resp. $\boldsymbol{\varepsilon}^{-}$) as the positive (resp. negative) part of the total strain obtained by projecting $\boldsymbol{\varepsilon}$ onto the space of all symmetric positive (resp. negative) semidefinite tensors with respect to the natural Frobenius norm, their model reads

$$
\begin{aligned}
& \psi_{0}^{ \pm}(\boldsymbol{\varepsilon})=\frac{1}{2} \lambda\langle\operatorname{tr} \boldsymbol{\varepsilon}\rangle_{ \pm}^{2}+\mu \boldsymbol{\varepsilon}_{ \pm} \cdot \boldsymbol{\varepsilon}_{ \pm} \\
& \boldsymbol{\tau}_{0}^{ \pm}(\boldsymbol{\varepsilon})=\lambda\langle\operatorname{tr} \boldsymbol{\varepsilon}\rangle_{ \pm} \mathbb{I}+2 \mu \boldsymbol{\varepsilon}_{ \pm}
\end{aligned}
$$

where contrary to the formulation (23) there is no more individual constitutive relation separately for the positive or the negative strain. Like the model of [10], the stress for a totally damaged element is negative semidefinite. We observe that the bracket operator $\langle\cdot\rangle_{ \pm}$applies to the trace of the total strain. If it is not the case as may be suggested by [8,27], i.e. when $\langle\operatorname{tr} \boldsymbol{\varepsilon}\rangle_{ \pm}$is replaced by $\operatorname{tr} \boldsymbol{\varepsilon}^{ \pm}$, then on one hand the decomposition $\psi_{0}^{ \pm}(\boldsymbol{\varepsilon})$ is no more a partition of the sound elastic energy (6) and on the other hand the usual stress identification (29) is no longer possible. In this case by definition the stress tensor applied to an arbitrary symmetric tensor $\mathbf{e}$ is given by

$$
\tau_{0}^{ \pm}(\boldsymbol{\varepsilon}) \mathbf{e}=\lambda \operatorname{tr} \boldsymbol{\varepsilon}^{ \pm} \operatorname{tr}\left(\frac{\partial \boldsymbol{\varepsilon}^{ \pm}}{\partial \boldsymbol{\varepsilon}}(\boldsymbol{\varepsilon}) \mathbf{e}\right)+2 \mu \varepsilon^{ \pm} \cdot \frac{\partial \boldsymbol{\varepsilon}^{ \pm}}{\partial \boldsymbol{\varepsilon}}(\boldsymbol{\varepsilon}) \mathbf{e}=\lambda \operatorname{tr} \boldsymbol{\varepsilon}^{ \pm} \operatorname{tr}\left(\frac{\partial \boldsymbol{\varepsilon}^{ \pm}}{\partial \boldsymbol{\varepsilon}}(\boldsymbol{\varepsilon}) \mathbf{e}\right)+2 \mu \boldsymbol{\varepsilon}^{ \pm} \cdot \mathbf{e}
$$

where the second equality follows thanks to the coaxiality among $\boldsymbol{\varepsilon}$ and $\boldsymbol{\varepsilon}^{ \pm}$. However the first term does not admit any furthermore simplification and the above unusual definition of the stress has to be adopted.

\subsection{Uniaxial traction and compression experiment}

Here we will investigate the theoretical behavior of the above outlined models under a very simple loading condition to illustrate their individual particularities. It can be understood that the underlying local damage model obtained by suppressing the gradient damage $\nabla \alpha_{t}$ in the dissipation energy density (10) represents the material behavior when no strain or damage localization appears. Hence some general properties of these tension-compression asymmetry models can be extracted under an academic homogeneous 3-dimensional uniaxial traction or compression experiment. Inertia is not essential for this analysis and will be neglected. We suppose that the stress tensor is of form $\tau_{t}=\tau_{33} \mathbf{e}_{3} \otimes \mathbf{e}_{3}$ corresponding to an imposed axial strain $\varepsilon_{33}=t$ viewed as a loading parameter. When $A$ is isotropic, the goal is to find the evolutions of the transversal strain $t \mapsto \varepsilon_{11}=\varepsilon_{22}$, the axial stress $t \mapsto \tau_{33}$ and the homogeneous damage $t \mapsto \alpha_{t}$. This amounts to solve the following system when the damage evolves $\dot{\alpha}_{t}>0$

$$
\begin{aligned}
& \tau_{11}(t)=\left(a\left(\alpha_{t}\right) \tau_{0}^{+}\left(\boldsymbol{\varepsilon}_{t}\right)+\tau_{0}^{-}\left(\boldsymbol{\varepsilon}_{t}\right)\right) \mathbf{e}_{1} \cdot \mathbf{e}_{1}=0, \\
& \frac{\partial \psi}{\partial \alpha}\left(\boldsymbol{\varepsilon}_{t}, \alpha_{t}\right)+w^{\prime}\left(\alpha_{t}\right)=0
\end{aligned}
$$

where $\boldsymbol{\varepsilon}_{t}=\varepsilon_{11}\left(\mathbf{e}_{1} \otimes \mathbf{e}_{1}+\mathbf{e}_{2} \otimes \mathbf{e}_{2}\right)+t \mathbf{e}_{3} \otimes \mathbf{e}_{3}$. The second equation (33b) is the local interpretation of the energy balance condition (17), see [12].

We remark that in order to solve (33) a particular set of damage constitutive laws also has to be chosen. Strictly speaking the functions $\alpha \mapsto a(\alpha)$ and $\alpha \mapsto w(\alpha)$ should influence the exact behavior of the tension-compression asymmetry models. Nevertheless we discover that the solutions obtained with two particular damage constitutive laws (PAMM) and (AT) share many qualitative properties.

The model of [25] has been already studied in this uniaxial traction and compression setting with the damage model (AT). The material undergoes a softening behavior both under tension or compression when a certain finite threshold $\tau_{0}^{ \pm}$is reached. The ratio between these two maximal stresses is given by

$$
-\frac{\tau_{0}^{-}}{\tau_{0}^{+}}=\sqrt{\frac{3}{2(1+v)}} \leq \sqrt{\frac{3}{2}} \approx 1.22
$$


which is not sufficient for applications to brittle materials where this factor can attain 10 . This ratio is the same when the damage constitutive law (PAMM) is used.

We then turn to the tension-compression separation proposed in [26]. Similar as it is to the model of [10] since both ones perform spectral decomposition of the total strain (with respect to two different inner products, though), their behavior under compression will be unexpectedly different. For the damage model of (PAMM), there exists as in the symmetric case a tensile $\tau_{0}^{+}$and a compressive $\tau_{0}^{-}$ stress threshold under which damage doesn't evolve

$$
\begin{aligned}
\tau_{0}^{+} & =\sqrt{\frac{(1+v)}{(1-v)(1+2 v)} w_{1} E,} \\
\tau_{0}^{-} & =-\sqrt{\frac{1+v}{2 v^{2}} w_{1} E} \rightarrow \infty \text { as } v \rightarrow 0 .
\end{aligned}
$$

It can be seen that the critical stress $\tau_{0}^{+}$increases with the Poisson ratio but stays bounded in tension. The compressive threshold $\tau_{0}^{-}$goes to infinity when $v$ is near zero, hence no damage will occur in this case. We use the tensile threshold $\tau_{0}^{+}$as well as its corresponding strain $\varepsilon_{0}^{+}$both evaluated at $v=0.2$ to normalize the results shown in Fig. 2 .
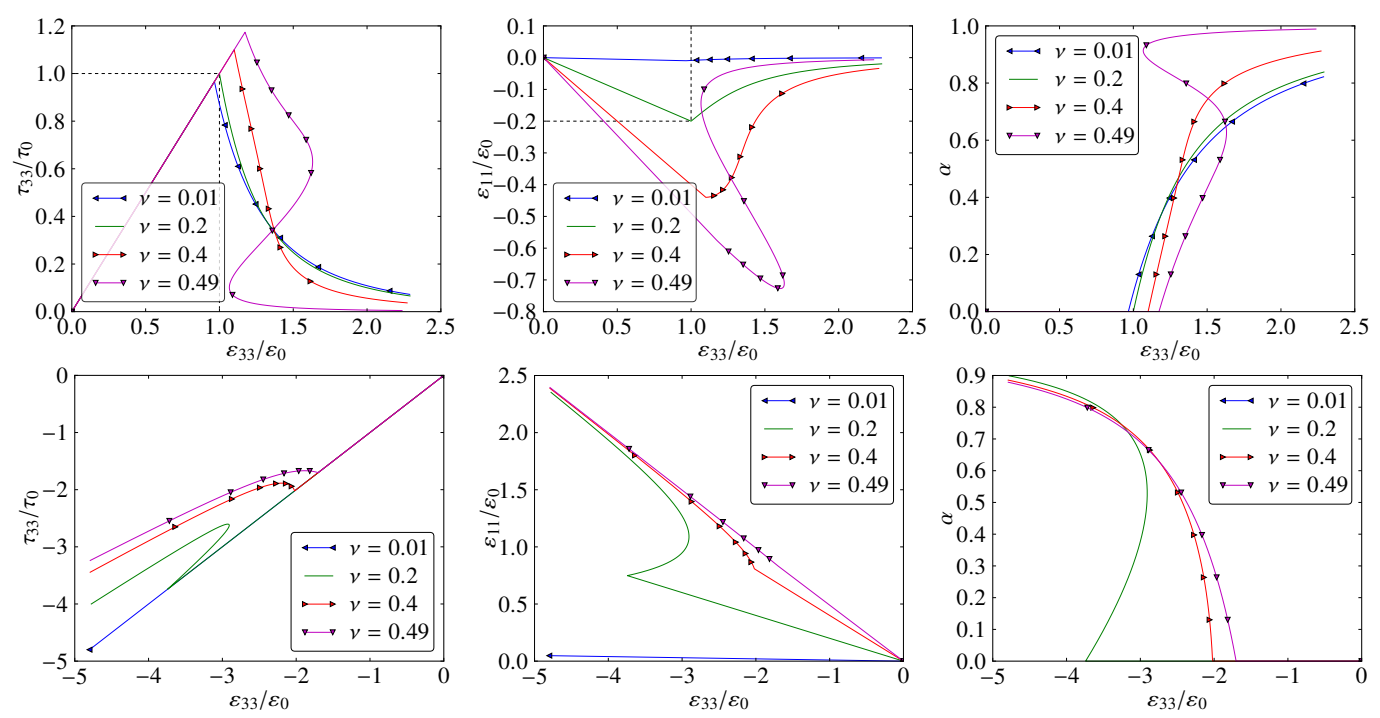

Figure 2. Uniaxial traction $\varepsilon_{33} \geq 0$ and compression $\varepsilon_{33} \leq 0$ experiment for the tension-compression asymmetry proposed in [26]. The damage constitutive law (PAMM) is used.

Under a uniaxial tensile loading, the material undergoes a classical softening behavior when the threshold stress is reached. For quasi-incompressible materials $v \approx \frac{1}{2}$ a snap-back is present and hence the evolution of the stress $\tau_{33}$ and the strain $\varepsilon_{11}$ may experience a temporal discontinuity. However this behavior is only limited to the law (PAMM) whereas for (AT) no snap-back is observed.

Unexpectedly, under compression the material may experience a two-phase softening-hardening (with an initial snap-back for $0 \leq v \leq 3 / 8$ limited to the (PAMM) case), while the damage increases. As $\alpha$ approaches 1, i.e. as the element becomes totally damaged, the uniaxial stress is not bounded and is given by $\tau_{33}=2 \mu \varepsilon_{33}$. Moreover, an apparent incompressible behavior is observed $\operatorname{tr} \boldsymbol{\varepsilon}_{t}=0$. These properties can be readily derived using the definitions (31). Due to a non-vanishing stress inside a completely damaged element, one may expect large diffusive "damage" for highly compressive zones. This may complicate the physical interpretation of the model of [26] in this situation.

In contrast, for any damage constitutive laws the model proposed in [10] does not permit any damage under uniaxial compression. The positive strain contributing to damage after projection (24) is given by $\boldsymbol{\varepsilon}_{0}^{+}=\left(\varepsilon_{11}+v \varepsilon_{33}\right)\left(\mathbf{e}_{1} \otimes \mathbf{e}_{1}+\mathbf{e}_{2} \otimes \mathbf{e}_{2}\right)$, which vanishes due to the uniaxial stress state $\boldsymbol{\tau}_{t}=\tau_{33} \mathbf{e}_{3} \otimes \mathbf{e}_{3}$ implying $\varepsilon_{11}=-v \varepsilon_{33}$. Under traction and when using the damage law (PAMM), a 
stress threshold under which no damage appears is given by

$$
\tau_{0}^{+}=\sqrt{\frac{(1-v)}{(1-2 v)(1+v)} w_{1} E} \rightarrow \infty \text { as } v \rightarrow \frac{1}{2}
$$

so cracks are impossible to appear for incompressible materials. We again use the tensile stress threshold $\tau_{0}^{+}$as well as its corresponding strain $\varepsilon_{0}^{+}$both evaluated at $v=0.2$ to normalize the results shown in Fig. 3. A classical softening behavior is observed after damage initiation. Analyses show that snapbacks are present for $v>(\sqrt{33}-1) / 16 \approx 0.3$. However it is only limited to the (PAMM) case.
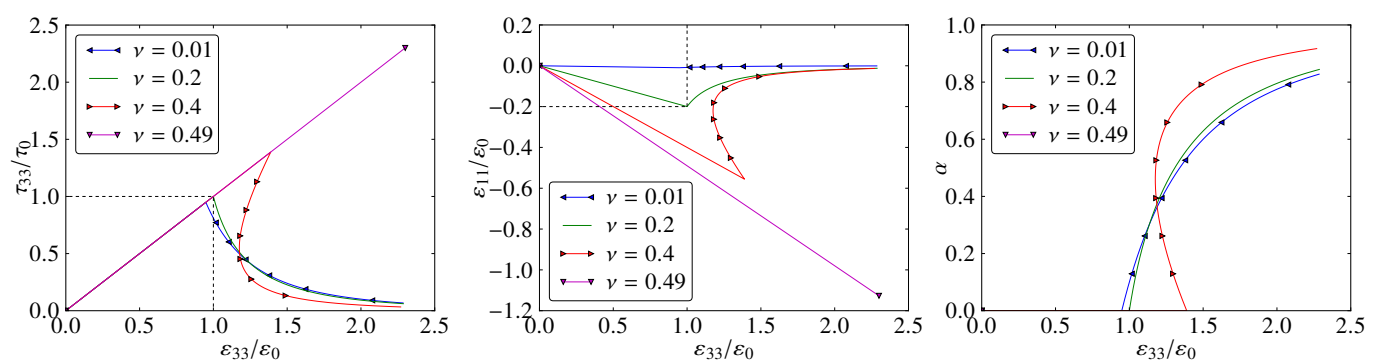

Figure 3. Uniaxial traction $\varepsilon_{33} \geq 0$ experiment for the tension-compression asymmetry proposed in [10]. The damage constitutive law (PAMM) is used.

\subsection{How to choose among different models}

Following the previous review and analyses of several existing models on tension-compression asymmetry, a natural question arises as to how to choose the best or the right one for a particular problem. If the variational formulation (24) is used, the problem can be reduced to choose a good convex cone $\mathcal{S}$ of the 2 nd-order symmetric tensors. As the elastic energy density split (22) influences both the displacement and the damage problems through the first order stability condition (16), these two aspects will be separately discussed.

- For the u-problem, the tension-compression asymmetry model is widely recognized to approximate the material non-interpenetration condition $[9,25,24]$. Together with the penalization of extreme compression (21) thanks to the Hencky's hyperelastic model (6), it should somehow avoid interpenetration of matter. However we would like to recall that this approximation is merely heuristic. Taking into account the actual non-interpenetration condition at finite strains in the sense of [28], i.e. local orientation preservation and global injectivity, is a difficult task both from a theoretical or numerical point of view, and hence is often merely checked a posteriori. Nevertheless we could expect that the tension-compression decomposition itself should depend on the local damage state and the damage gradient $\nabla \alpha_{t}$ approximating the local crack normal in the reference frame. A better elastic energy density split of (22) could be

$$
\psi(\boldsymbol{\varepsilon}, \alpha, \nabla \alpha)=a(\alpha) \psi_{0}^{+}(\boldsymbol{\varepsilon}, \alpha, \nabla \alpha)+\psi_{0}^{-}(\boldsymbol{\varepsilon}, \alpha, \nabla \alpha) .
$$

When the crack is created, the elastic energy split itself should become orientation dependent so that only non-positive normal stress can be applied on crack lips if friction is not considered. The extension to this kind of energy splits is being considered in [29].

- For the $\alpha$-problem, the decomposition (22) directly controls the type of strain or stress state which initiates and produces further damage: deviatoric part in [9] or in [11] under compression and positive principal values in [26, 10]. We share the remark given in [24] that only experiments conducted with real materials can determine or identify a good model. We thus regard the elastic energy split (22) or the convex cone $\mathcal{S}$ as another independent material 
property or parameter characterizing the microstructure. For rocks or stones the deviatoric model may predict realistic crack path, however for more brittle materials such as glass, models based on a spectral decomposition may be more suitable.

\section{NUMERICAL IMPLEMENTATION}

This section is devoted to numerical implementation of Model 1 applied to explicit dynamic situations. The two damage constitutive laws (AT) and (PAMM) will be used. The elastic energy density split (22) is also adopted to take into account tension-compression asymmetry. Our implementation can be considered as a large displacement extension of the previous work of $[11,30,1]$ on quasi-static and dynamic phase-field like models for fracture. Similarily, only the irreversiblity condition and the variational inequality (16) will be effectively implemented, which amounts to solve numerically the classical wave equation (19) and the minimality principle for damage (20) at every time step. However it is shown in [31] that the time-discrete numerical model will also balance energy as required in (17), when the time increment becomes small. Their constructive proof makes use of the implicit Euler scheme used in [30], however our experience suggests the same for the explicit central difference scheme as we describe below.

In this contribution the spatial and temporal discretization is as usual decoupled and will be discussed below separately. Space-time finite element methods will exploit fully the variational nature of the formulation (16) and can be considered as a possible improvement in the future.

\subsection{Spatial discretization}

Classical finite element method is used to discretize in space the displacement $\mathbf{u}_{t}$ and the damage field $\alpha_{t}$ based on a same mesh $\Omega_{h}$. Usual geometrical element types can be used: triangular or quadrilateral elements in 2-d; tetrahedral or hexagonal elements in 3-d, to name just a few. It should be ideally unstructured and uniform in mesh sizes otherwise some directions may be preferred when cracks propagate [11]. The typical element size $h$ of the mesh should be comparable and preferably smaller with respect to the internal length $\ell$ in order to calculate correctly the damage dissipation energy (4) and the material response inside the crack process zone of order $O(\ell)$, see [6].

Displacement problem The displacement $\mathbf{u}_{t}$ and the damage field $\alpha_{t}$ will be both discretized with linear isoparametric finite elements as nodal vectors [11, 20]. Inside an arbitrary element $\Omega_{\mathrm{e}} \in \Omega_{h}$, we thus have

$$
\begin{aligned}
& \mathbf{u}_{t}(\mathbf{x})=\mathbf{N}(\mathbf{x}) \underline{\mathbf{u}} \quad \text { and } \quad \nabla^{\mathrm{s}} \mathbf{u}_{t}(\mathbf{x})=\mathbf{B}(\mathbf{x}) \underline{\mathbf{u}}, \\
& \alpha_{t}(\mathbf{x})=\mathbf{N}_{\alpha}(\mathbf{x}) \underline{\alpha} \quad \text { and } \quad \nabla \alpha_{t}(\mathbf{x})=\mathbf{B}_{\alpha}(\mathbf{x}) \underline{\alpha}
\end{aligned}
$$

where $\mathbf{N}$ and $\mathbf{B}$ are respectively the interpolation and differentiation matrices applied on local nodal vectors $\underline{\mathbf{u}}$ and $\underline{\alpha}$ specific to the element $\Omega_{\mathrm{e}}$. The Hencky logarithmic strain (5) depends nonlinearly on the displacement vector and will be denoted by

$$
\boldsymbol{\varepsilon}_{t}(\mathbf{x})=\mathbf{h}(\mathbf{x}, \underline{\mathbf{u}}) .
$$

Its effective calculation will be detailed in Sect. 4.2. In explicit dynamics linear elements are largely preferred due to their low computational cost and an easily obtained diagonal lumped mass matrix. According to [6], linear elements perform equally well compared to higher-order elements in terms of $\Gamma$-convergence. Finally this P1-P1 finite element discretization is not forbidden according to [32].

Owing to the explicit Newmark scheme to be described in Sect. 4.2, at the beginning of every time step the current configuration $\phi_{t}(\Omega)$ is already calculated from the last iteration. Hence the Eulerian elastodynamic equation (19) will be naturally solved on the deformed mesh obtained by constantly updating the mesh coordinates $\mathbf{x}_{t}=\mathbf{x}+\mathbf{u}_{t}(\mathbf{x}) \in \Omega_{t}$. This is referred to the updated Lagrangian formulation [17]. However in other general implicit cases the dynamic or static equilibrium can only be prescribed in the last known configuration. The wave equation (19) after discretization in space 
reads

$$
\mathbf{M} \underline{\ddot{u}}=\mathbf{F}_{\text {ext }}-\mathbf{F}_{\text {int }}(\underline{\mathbf{u}}, \underline{\alpha})
$$

with $\mathbf{M}$ the classical lumped mass matrix, $\mathbf{F}_{\text {ext }}$ the external force vector corresponding to the potential (3) and $\mathbf{F}_{\text {int }}$ the internal force vector assembled from the elementary vectors given by

$$
\begin{aligned}
& \mathbf{F}_{\text {int }}^{\mathrm{e}}=\int_{\Omega_{\mathrm{e}}} \mathbf{B}^{\top} \boldsymbol{\sigma}\left(\mathbf{h}(\cdot, \underline{\mathbf{u}}), \mathbf{N}_{\alpha} \underline{\boldsymbol{\alpha}}\right) \\
& =\int_{\Omega_{\mathrm{e}}} \mathbf{B}^{\top}\left(a\left(\mathbf{N}_{\alpha} \underline{\boldsymbol{\alpha}}\right) \boldsymbol{\sigma}_{0}^{+}(\mathbf{h}(\cdot, \underline{\mathbf{u}}))+\sigma_{0}^{-}(\mathbf{h}(\cdot, \underline{\mathbf{u}}))\right) .
\end{aligned}
$$

For simplex finite elements (triangular and tetrahedral elements) the Jacobian of the transformation (between the reference and the physical elements) as well as the differentiation matrix $\mathbf{B}$ are constant, thus (37) can be integrated exactly using an effective stress

$$
\mathbf{F}_{\text {int }}^{\mathrm{e}}=\left|\Omega_{\mathrm{e}}\right| \mathbf{B}^{\top} \sigma_{\text {eff }}=\left|\Omega_{\mathrm{e}}\right| \mathbf{B}^{\top}\left(a_{\mathrm{eff}} \boldsymbol{\sigma}_{0}^{+}+\boldsymbol{\sigma}_{0}^{-}\right)
$$

with $a_{\text {eff }}$ the effective stiffness degradation

$$
a_{\mathrm{eff}}=\frac{1}{\left|\Omega_{\mathrm{e}}\right|} \int_{\Omega_{\mathrm{e}}} a\left(\mathbf{N}_{\alpha} \underline{\boldsymbol{\alpha}}\right) .
$$

For tensor product finite elements, a loop on the Gauss points is necessary and our experience suggests that 4 Gauss points for quadrilateral elements and 8 for hexagonal elements are sufficient. We also note that in explicit dynamics a residual stiffness $k_{\text {res }}$ is not needed in the stiffness degradation function as no matrix inversion is needed, contrary to the implicit cases $[11,3]$.

Damage problem According to (20), the damage minimality condition is naturally formulated in the initial reference configuration. From a physical point of view, this corresponds to the fact that the damage dissipation energy is destined to measure the length or the area of cracks defined in the reference configuration. Technically the concerned energies can be written in the deformed mesh (at the expense of additional unpleasant $\mathbf{F}_{t}$ and $J_{t}$ terms), however it is not necessary [17]. Using the damage constitutive laws (AT) and (PAMM), the total damageable energy $\mathcal{E}+\mathcal{S}$ is quadratic with respect to the damage vector $\underline{\alpha}$ and (20) after spatial discretization reads

$$
q_{\underline{\mathbf{u}}}(\underline{\boldsymbol{\alpha}}) \leq q_{\underline{\mathbf{u}}}(\underline{\boldsymbol{\beta}}) \text { for all } \underline{\boldsymbol{\beta}} \text { that } 0 \leq \underline{\boldsymbol{\alpha}} \leq \underline{\boldsymbol{\beta}} \leq 1
$$

with the quadratic function defined by

$$
q_{\underline{\mathbf{u}}}(\underline{\boldsymbol{\alpha}})=\frac{1}{2} \underline{\boldsymbol{\alpha}}^{\top} \mathbf{H}(\underline{\mathbf{u}}) \underline{\boldsymbol{\alpha}}-\mathbf{b}(\underline{\mathbf{u}})^{\top} \underline{\boldsymbol{\alpha}} .
$$

The Hessian matrix $\mathbf{H}$ and the second member vector $\mathbf{b}$ depend solely on the current deformation state $\underline{\mathbf{u}}$ and hence are constant during the solving process of the damage problem. Their exact forms depend on the damage constitutive law used.

\subsection{Temporal discretization}

Given an arbitrary discretization $\left(t^{n}\right)$ of the time interval of interest $I$ where the superscript $n$ denotes a quantity evaluated at the $n$-th time step, our objective here is to solve the spatially discretized wave equation (36) coupled with the crack minimality condition (39) at these steps. In this contribution we consider dynamic fracture problems in brittle materials under impact-type loadings. In general the time scale involved is typically of order $O(1 \mathrm{~ms}) \ll O(1 \mathrm{~s})$, thus in absence of a costly matrix inversion the explicit Newmark scheme with a lumped mass matrix is very suitable for this kind of situations. It is a special case of the $\beta$-Newmark family [33] with $\beta=0$ and hence is second-order accurate, symplectic which implies energy conservation but unfortunately conditionally stable. After spatial discretization the constraint prescribed on the current time increment $\Delta t$ is often determined 
by the CFL condition $\Delta t<\Delta t_{\mathrm{CFL}}=\min (h / c)$ where $h$ is the mesh size, $c$ is the material sound speed and the smallest value is chosen among all elements. This is not a very inconvenient feature in our application since in presence of high geometrical and material nonlinearities even unconditionally stable implicit schemes need a small time increment comparable to $\Delta t_{\mathrm{CFL}}$. In the calculation of the material sound speed, current damage state as well as the tension-compression split formulation is taken into account. Thus a totally damaged element under tension does not penalize the total computational time.

We note that after temporal discretization (39) reads

$$
q_{\underline{\mathbf{u}}^{n}}\left(\underline{\boldsymbol{\alpha}}^{n}\right) \leq q_{\underline{\mathbf{u}}^{n}}(\underline{\boldsymbol{\beta}}) \text { for all } \underline{\boldsymbol{\beta}} \text { that } 0 \leq \underline{\boldsymbol{\alpha}}^{n-1} \leq \underline{\boldsymbol{\beta}} \leq 1
$$

where the Hessian matrix and the second member vector in (40) are evaluated at $\underline{\mathbf{u}}^{n}$. It can be translated to an effective minimization of the quadratic function $q$ under the constraint that $\underline{\alpha}^{n}$ is pointwise non-decreasing with respect to its previous value. In absence of the temporal derivative of the damage field $\dot{\alpha}_{t},(41)$ is not a genuine time evolution problem as the sole time dependence is introduced via the irreversibility condition. The current damage $\underline{\alpha}^{n}$ can be accurately calculated as long as the current deformation state $\underline{\mathbf{u}}^{n}$ is known.

In the time-continuous model the wave equation (19) and the damage minimality condition (20) are coupled in the first-order stability principle (16). After discretization $\underline{\mathbf{u}}$ and $\underline{\alpha}$ evaluated at the last and current iterations are in general involved in a non-explicit fashion. We can use a monolithic scheme which solves simultaneously at a time step the above two subproblems using a Newton-type nonlinear solver $[1,3]$. A staggered scheme which decouples algorithmically the wave equation and the energy minimization problem is also widely used [30,2]. In our case it turns out that the explicit Newmark scheme automatically decouples the time evolution system in $(\underline{\mathbf{u}}, \underline{\boldsymbol{\alpha}})$ and the two subproblems can be independently solved one from the other at every time step. It is due to the fact that the current acceleration $\underline{\ddot{u}}^{n}$ can be obtained based on the current known deformation state $\underline{\mathbf{u}}^{n}$ and the current damage $\underline{\alpha}^{n}$ calculated from $\underline{\mathbf{u}}^{n}$. The time-stepping scheme can be summarized in Algorithm 1. It is known as the Velocity Verlet implementation since velocities at mid-steps appear and all quantities at time $t^{n}$ are obtained after the $n$-th time step and can be used for post-processing. A bound-constrained minimization problem appears in every time iteration of the elastodynamic equation, and thus its efficient implementation is crucial. We observe that the initial damage is recomputed $\underline{\alpha}^{-1} \mapsto \underline{\alpha}^{0}$ in the step 2 . The role of $\underline{\alpha}^{-1}$ is to bring some a priori knowledge of the damage field resulting from a previous calculation or more frequently to represent an initial crack $\underline{\alpha}^{-1}=1$ on $\Gamma_{0}$. The initial step 2 thus renders it compatible with the initial displacement condition and the energy minimization structure.

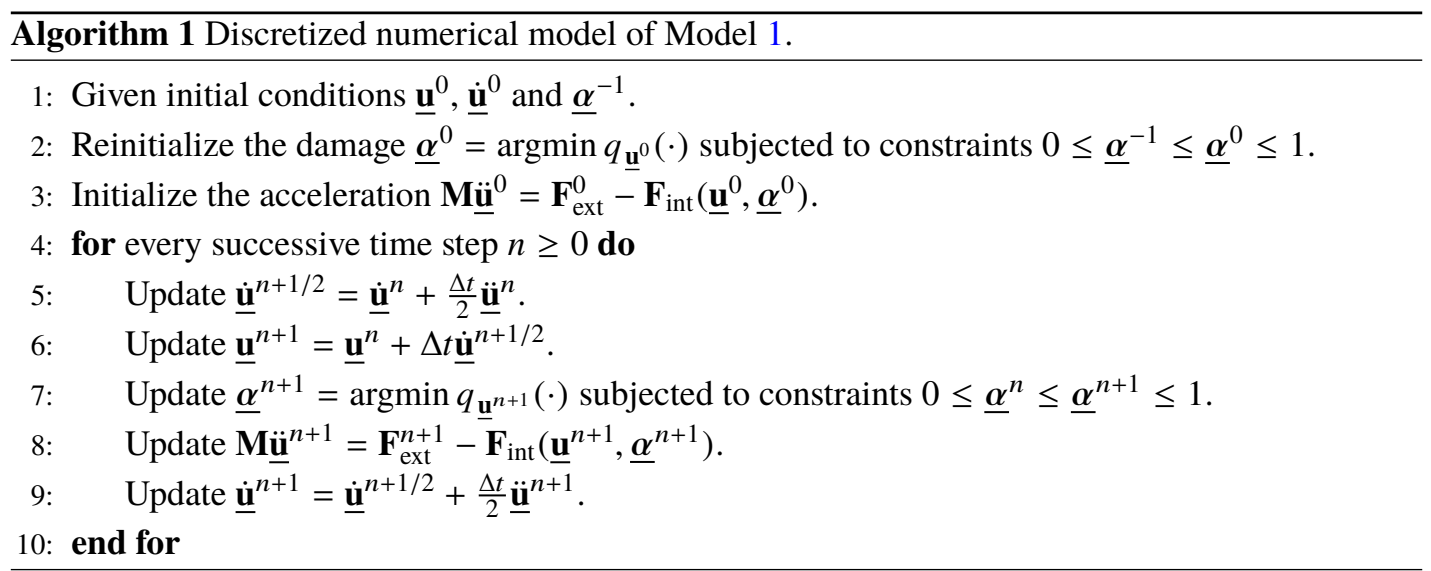

We end this section on temporal discretization by the numerical calculation of the Hencky logarithmic strain $\mathbf{h}_{t}$ defined in (5). Although the polar decomposition is indeed unnecessary by remarking that $\log \mathbf{V}_{t}=\frac{1}{2} \log \mathbf{B}_{t}$, spectral decomposition of the left Cauchy-Green tensor $\mathbf{B}_{t}=\mathbf{F}_{t} \mathbf{F}_{t}^{\top}$ is unavoidable. In this work we use an approximation of the Hencky strain based on the following 
remarkable property established in [18]: within a proper corotational frame the rate of the Hencky strain equals the stretching tensor. Combined with the time-stepping scheme in Algorithm 1 an increment of the Hencky strain can be given by $\Delta \mathbf{h}^{n} \approx \nabla^{\mathrm{s}} \Delta \mathbf{u}^{n}$ where $\Delta \mathbf{u}^{n}=\mathbf{u}^{n}-\mathbf{u}^{n-1}$ is referred to the current configuration. To have better accuracy, a second-order approximation based on the incremental Almansi strain is used

$$
\Delta \mathbf{h}^{n} \approx \frac{1}{2}\left(\nabla \Delta \mathbf{u}^{n}+\nabla^{\top} \Delta \mathbf{u}^{n}-\left(\nabla^{\top} \Delta \mathbf{u}^{n}\right)\left(\nabla \Delta \mathbf{u}^{n}\right)\right) .
$$

Finally the current Hencky strain is incremented $\mathbf{h}^{n}=\mathbf{h}^{n-1}+\Delta \mathbf{h}^{n}$.

\subsection{Implementation}

Contrary to a pure explicit dynamic calculation, the presence of an implicit damage problem (41) calls for a parallel linear algebra backend for manipulation of sparse matrices and vectors. As in the work of [20], the library PETSc [34] is adopted since it provides also an efficient numerical scheme GPCG initially proposed in [35], designed for quadratic bound-constrained minimization problems. It consists of several gradient projections to identify the active nodes, i.e. those either $\underline{\alpha}^{n}=\underline{\alpha}^{n-1}$ or $\underline{\alpha}^{n}=1$. Then it applies the preconditioned conjugate gradient (PCG) method to minimize an unconstrained reduced problem of the free variables, i.e. those satisfying $\underline{\boldsymbol{\alpha}}^{n-1}<\underline{\boldsymbol{\alpha}}^{n}<1$. The incomplete Cholesky factorization preconditioner is applied block-wise onto each decomposed subdomain. Our simulation results demonstrate that this scheme is robust and efficient. Computational load is also well balanced [36] in parallel computations based on domain decomposition.

Algorithm 1 has been fully implemented in EUROPLEXUS, an explicit dynamics program dedicated to transient phenomena involving fluid-structure interaction [37]. Meanwhile an opensource implementation of the model under small displacement hypothesis is also available [38]. It is based on the FEniCS Project [39] for automated solution of PDE's.

\section{SIMULATION RESULTS}

\subsection{Dynamic crack branching}

We will first study the dynamic crack branching problem for a 2-d plane stress plate under constant pressure applied on its upper and lower boundaries. This particular problem has already been investigated within the phase-field community $[1,3]$ where the numerical convergence aspect as well as the physical insight into the branching mechanism are analyzed. Here we will mainly focus on the computational efficiency as well as the possible use of several damage constitutive laws to approximate fracture.

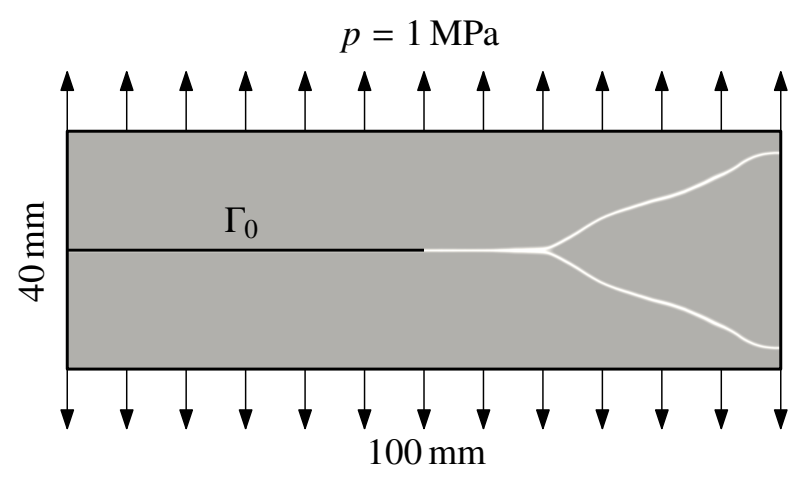

Figure 4. Geometry and loading conditions for the dynamic crack branching problem. Damage field $\alpha_{t}$ at $t=8 \times 10^{-5} \mathrm{~s}$ ranging from 0 (gray) to 1 (white). 
The geometry as well as the loading conditions are depicted in Fig. 4. Due to symmetry only the upper half part is modeled. The initial crack $\Gamma_{0}$ is introduced via an initial damage field $\alpha^{-1}$. Material parameters are borrowed from [1] where the internal length $\ell$ is set to $0.25 \mathrm{~mm}$. We use a structured quadrilateral elements of equal discretization spacing $h \approx 0.045 \mathrm{~mm}$ in both directions achieving approximately 1 million elements. An unstructured mesh should be in general preferred. However the original analysis on mesh-induced anisotropy is conducted on structured triangular elements [40]. Furthermore the numerical study of [41] shows that the crack direction is insensitive to the orientation of a structured quadrilateral grids. We firstly use the damage constitutive law (PAMM). The symmetric tension-compression formulation is also adopted. This choice is justified by an $a$ posteriori verification of non-interpenetration of matter. The simulation result is illustrated in Fig. 4 by the damage field $\alpha_{t}$ at $t=8 \times 10^{-5} \mathrm{~s}$ ranging from 0 (gray) to 1 (white). Similar contours have been obtained in $[1,3]$.

A strong scaling analysis is conducted for several processor cores NP in the cluster Aster5 [42] provided by the Electricite de France. We have verified that all simulations give nearly the same results in terms of global energy evolution and field contours. The difference of the elastic energy at $t=8 \times 10^{-5} \mathrm{~s}$ is within $0.2 \%$ between the sequential and the parallel $\mathrm{NP}=16$ cases, which may be due to floating point arithmetic and different setting of preconditioners. The scaling results are given in Fig. 5. The calculation time is partitioned into 4 items: the "elastodynamics" part related to the solving of (36), the "damage assembly" part where the global Hessian matrix $\mathbf{H}$ and the second member $\mathbf{b}$ is constructed, the "damage solving" part where (41) is solved and the "communication" part corresponding to the data exchange among processors. The maximum value among all processors are used. Quasi-ideal scaling is observed for the total computational time. The proportion of the "elastodynamics" and the "damage assembly" parts are decreasing, due to the increase of the "communication" overhead reaching $15 \%$ with 16 cores and becoming comparable to that of the "damage solving".

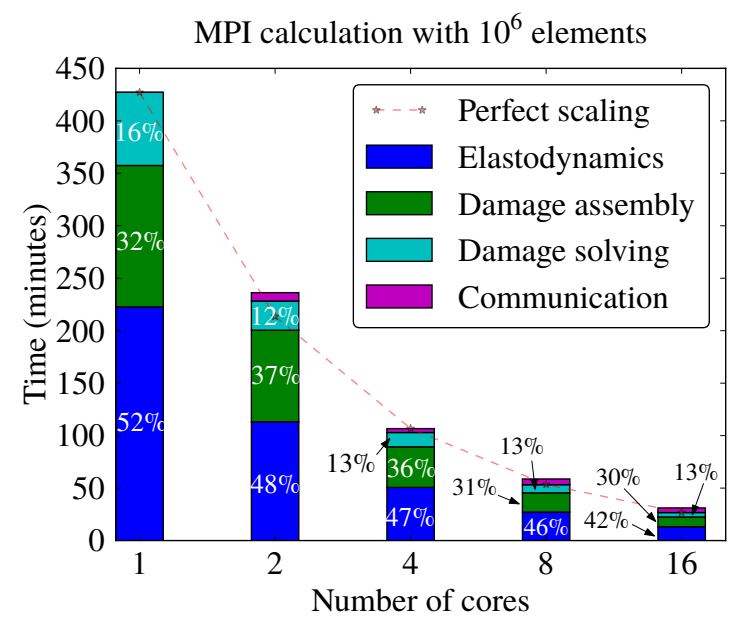

Figure 5. Strong scaling results for the dynamic crack branching problem with 1 million elements.

We remark that the quadratic bound-constrained minimization problem (41) solved by the GPCG scheme implemented in PETSc is not very costly and represents in sequential and parallel calculations only $13 \%$ of the total computational time. In the phase-field literature the damage problem is often solved by an unconstrained minimization of (41) corresponding to a linear system

$$
\mathbf{H} \underline{\alpha}=\mathbf{b} .
$$

To reinforce irreversibility, either the damage driving term is replaced by a history field [2, 1], or (42) is followed by an a posteriori projection in the admissible space, see [9]. However, it should be borne in mind that the above computationally-appealing strategy only applies to the damage constitutive law (AT), where the solution of (42) lies necessarily between 0 and 1 and the objective function $q$ is indeed 
quadratic with respect to $\alpha_{t}$. Otherwise a specific numerical scheme for bound-constrained problems is needed. Nevertheless we would like to point out that the GPCG solver is extremely efficient even compared to the above strategy consisting of only one linear equation. The same crack branching analysis is conducted using (AT) using a same internal length $\ell=0.25 \mathrm{~mm}$ and the results obtained with the GPCG solver and the above a posteriori projection method are compared. In the latter case the same PCG method is employed to solve (42). The results are slightly different as expected, since the projection method does not solve exactly the full minimization problem (41). To compare their relative computational costs, the time consumed in damage solving is separately normalized by that corresponding to the elastodynamic problem in Tab. I. Opposed to what is suggested by [25], the use of a bound-constrained minimization solver implies a relative computational cost only $27 \%$ higher than a traditional linear solver. This can be seen in the normalized histogram of CG iterations per time step illustrated in Fig. 6. We recall that each CG iteration implies a matrix-vector multiplication, the most costly part of the algorithm. When only one linear system is to be solved in the a posteriori projection method, approximately $20 \mathrm{CG}$ iterations are needed in $35 \%$ of all time steps. When the GPCG solver is used, we observe that the histogram is more spread out and more than $50 \mathrm{CG}$ iterations may be needed for some time steps. Nevertheless the distribution is more concentrated around 10 to 30 iterations.

Table I. Relative damage-solving cost normalized by the time devoted to the elastodynamic part during a parallel calculation NP $=16$. The damage constitutive law (AT) is used. Comparison between the GPCG solver and the a posteriori projection method.

\begin{tabular}{lll}
\hline & CG + projection & GPCG \\
\hline Damage-solving cost & $50 \%$ & $77 \%$ \\
\hline
\end{tabular}

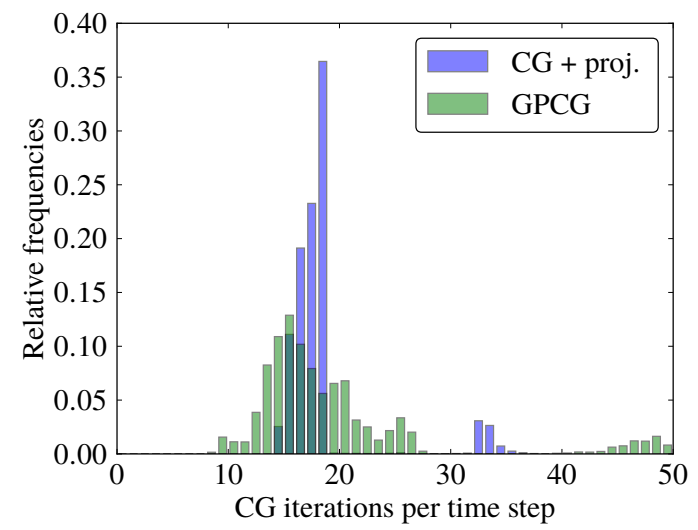

Figure 6. Normalized histogram of CG iterations per time step. The damage constitutive law (AT) is used. Comparison between the GPCG solver and the a posteriori projection method.

We then turn to the choice of different damage constitutive laws from a computational and physical point of view. We take the simulation results using (PAMM) as a reference and compare it with the widely used damage constitutive law (AT) in phase-field modeling of fracture. In the latter case, two values of the material internal length $\ell$ have been chosen: one corresponding to the same value $\ell=0.25 \mathrm{~mm}$ as used in the (PAMM) case, the other corresponding to a same maximal tensile stress as used in the $(\mathrm{PAMM})$ case, which gives $\ell \approx 0.07 \mathrm{~mm}$. We recall from [11] that the maximal stress than can be supported by a gradient damage material is given by

$$
\sigma_{\mathrm{m}}= \begin{cases}\sqrt{\frac{3 G_{\mathrm{c}} E}{8 \ell}} & (\mathrm{PAMM}) \text { case } \\ \frac{3 \sqrt{3}}{16} \sqrt{\frac{G_{\mathrm{c}} E}{\ell}} & \text { (AT) case, }\end{cases}
$$


which determines the internal length as long as the material toughness and the Young's modulus are fixed. The same GPCG solver is used and the relative damage-solving costs separately normalized by the time devoted to the elastodynamic part are reported in Tab. II. We remark that the use of the constitutive law (PAMM) or a smaller internal length $\ell$ reduces significantly the damage-solving time. A viable explanation is given as follows. The theoretical 1-d damage profile of (AT) corresponds to an exponential function without a finite support $[6,26]$. The damage band $2 D$, i.e. in which $\alpha_{t}>0$, is much wider than the (PAMM) case where $D=2 \ell$. Consequently, less active nodes are present and the GPCG solver identifies much more free nodes for the (AT) case, which induces a bigger linear system to be solved. Similarly, a reduction of the material internal length may imply finer mesh along the crack path, however the damage is more concentrated and the relative solving cost is decreased.

Table II. Relative damage-solving cost normalized by the time devoted to the elastodynamic part during a parallel calculation NP $=16$. The GPCG solver is used. Comparison between different constitutive laws.

\begin{tabular}{ll}
\hline & Damage-solving cost \\
\hline (PAMM) & $32 \%$ \\
(AT) with a same $\ell$ & $77 \%$ \\
(AT) with a same $\sigma_{\mathrm{m}}$ & $36 \%$ \\
\hline
\end{tabular}

The damage field $\alpha_{t}$ at $t=8 \times 10^{-5} \mathrm{~s}$ obtained with the constitutive law (AT) is illustrated in Fig. 7. Recall that the same mesh with $h=0.05 \mathrm{~mm}$ is used and should be sufficient for both calculations. Compared to Fig. 4 obtained with (PAMM), the transition area where $0<\alpha_{t}<1$ is more pronounced especially in Fig. 7a, conforming to the above discussions on the damage band. Another reason behind a relatively large zone with intermediate damage values is due to the different stress-strain behavior of these two constitutive laws during a homogeneous traction experiment [11]. In the (PAMM) case the material possesses a purely elastic domain and damage doesn't evolve as long as the maximal stress in (43) is not reached. Then the material follows a classical softening behavior as damage grows from 0 to 1 . However for the constitutive law (AT) widely used in phasefield modeling, damage evolves the instant when the material is subjected to external loadings. An elastic domain is absent and stress-hardening is observed within the damage interval $\left[0, \frac{1}{4}\right]$, as is already been reported by [1,3]. In this case the phase-field $\alpha_{t}$ loses its physical interpretation as damage, and hence correctly handling and interpreting crack healing is not trivial [3].

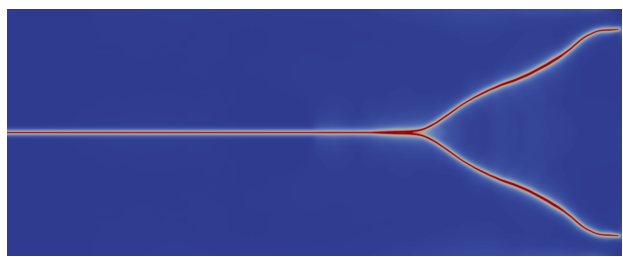

(a) (AT) with $\ell=0.25 \mathrm{~mm}$.

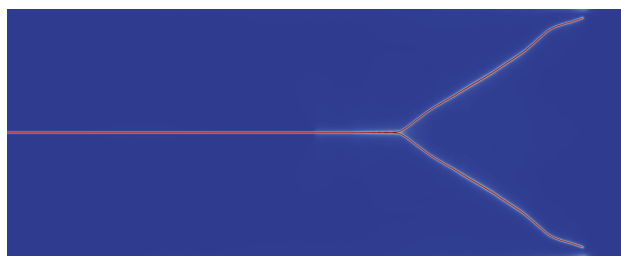

(b) (AT) with $\ell \approx 0.07 \mathrm{~mm}$.

Figure 7. Damage field $\alpha_{t}$ at $t=8 \times 10^{-5} \mathrm{~s}$ ranging from 0 (blue) to 1 (red) for the dynamic branching problem. Comparison between two internal lengths with the same constitutive model (AT).

Furthermore, this peculiar behavior of the constitutive law (AT) also contributes to an overestimation of the dissipated energy, as is noted in [1,43]. The energy evolution in this dynamic crack branching problem is given in Fig. 8. It is observed that the (AT) law produces a dissipated energy much bigger than the (PAMM) case, although according to Fig. 7 the damage fields are similar.

As can be seen from Fig. 7 and 8, apparently the results obtained with the same internal length $\ell$ resembles better the (PAMM) calculation in Fig. 4, even though it corresponds to a smaller maximal stress than the latter case. It should be reminded that $\ell$ does not play merely the role of determination of the maximal stress as in (43). From [12], this parameters also contributes qualitatively to the separation of the outer linear elastic fracture mechanics problem and the inner crack tip problem in 
Solid lines: elastic, dashed lines: dissipated

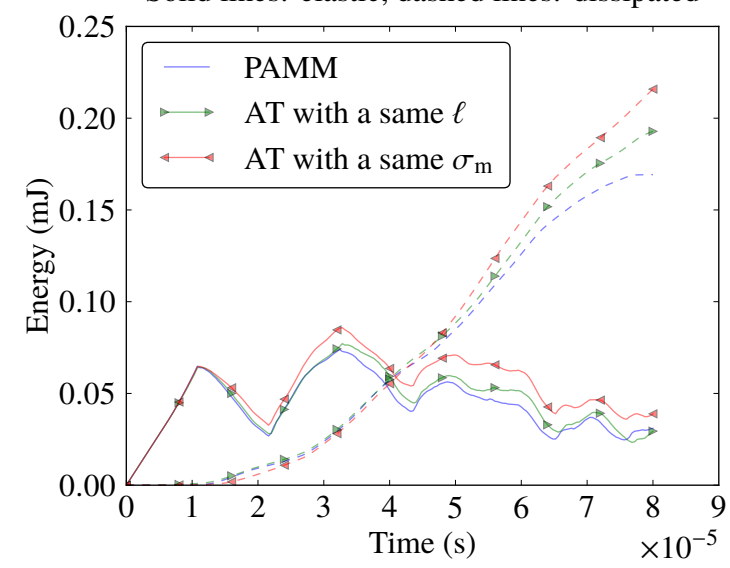

Figure 8. Energy evolution for the dynamic crack branching problem obtained with several constitutive laws.

an asymptotic context. A smaller internal length implies a wider region outside the crack where the fracture mechanics theory may apply. Meanwhile, a size effect is also introduced via this internal length as it influences the stability of a structure [44]. We admit that the choice of this parameter is not a simple one and may constitute one of the difficulties in phase-field modeling of fracture problems.

\subsection{Edge-cracked plate under shearing impact}

We then consider a pre-notched two-dimensional plane strain plate impacted by a projectile. In the dynamic fracture community this is often referred to the Kalthoff-Winkler experiment reported by e.g. [45] where a failure mode transition from brittle to ductile fracture is observed for a high strength maraging steel when the impact velocity is increased. Due to symmetry, only the upper half part of the plate will be considered. The geometry and the boundary conditions for the reduced problem are described in Fig. 9. As in [1, 2], the projectile impact is modeled by a prescribed

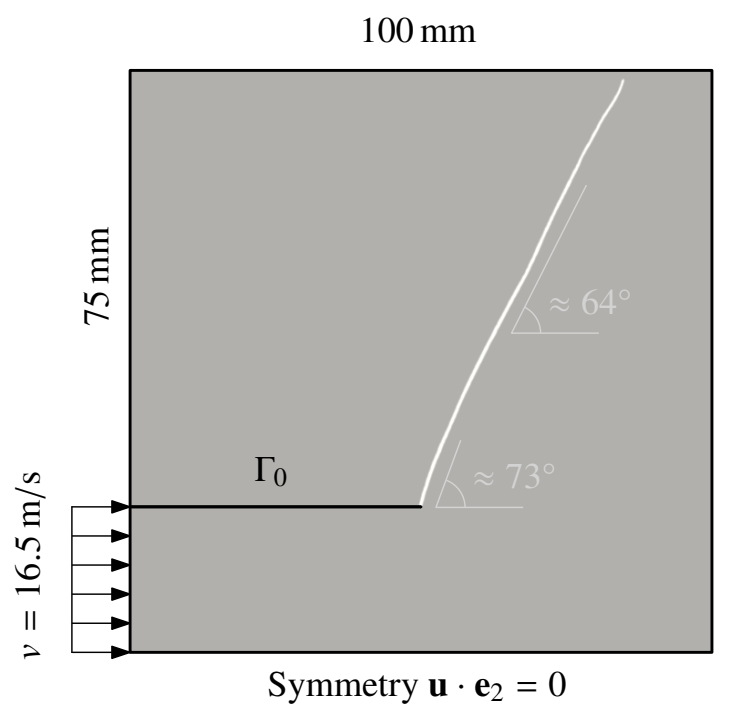

Figure 9. Geometry and boundary conditions for the edge-cracked plate under shearing impact problem.

Damage field $\alpha_{t}$ at $t=8 \times 10^{-5} \mathrm{~s}$ ranging from 0 (gray) to 1 (white).

velocity with an initial rise time of $1 \times 10^{-6} \mathrm{~s}$ to avoid acceleration shocks. The material parameters 
are borrowed from [1] except that the internal length $\ell$ is set to $0.2 \mathrm{~mm}$. An unstructured and uniform triangular mesh with $h \approx 0.1 \mathrm{~mm}$ is used, arriving at approximately 3 million elements. Due to a lower computational cost and a more brittle material behavior, the damage constitutive law (PAMM) is used for this simulation.

As a reference, we use the elastic energy split proposed in [10] where the positive semidefinite part of the total strain will contribute to damage. The initial crack is introduced via a real notch in the geometry. A similar strong scaling curve as Fig. 5 is obtained with up to 32 cores. Due to the additional spectral decomposition, the "damage assembly" phase represents now approximately $50 \%$ of the total computational time while the "damage solving" still accounts for only 10\%. The actual computation of the eigenvalues and eigenvectors of a $3 \times 3$ symmetric matrix is performed by a robust and efficient semi-analytic algorithm described in [46]. The damage field $\alpha_{t}$ at $t=8 \times 10^{-5} \mathrm{~s}$ is depicted in Fig. 9 , obtained with an imposed impact speed $v=16.5 \mathrm{~m} / \mathrm{s}$. The initial and average propagation angles are in good agreement with the experimental results and other phase-field simulations $[1,2]$ based on the damage constitutive law (AT) and the tension-compression asymmetry formulation proposed by [26].

If the initial crack $\Gamma_{0}$ is modeled via an initial damage field $\alpha^{-1}$, as for the previous dynamic crack branching example, no crack propagation is observed and the structures behaves as if the crack does not exist, i.e. the crack closure phenomenon. The horizontal displacement $u_{x}$ obtained in both cases at $t \approx 2.4 \times 10^{-5} \mathrm{~s}$ when the real notch case starts to propagate is presented in Fig. 10. In the real notch case, contact condition is not prescribed on the initial crack lips distanced by a finite height $\approx h$ in the geometry. As can be checked from Fig. 10a, no material interpenetration happens and waves propagate in the plate through the lower impacted edge. However in the initial damage case, possible normal compressive stresses can be transferred to the upper part of the plate, via the tension-compression asymmetry model [10] which simulates a crack clousure. However our simulation illustrates that this model also prohibits tangential relative movement along the crack lips, and a perfect adhesion (no-slip condition) is observed, i.e. exactly the opposite situation compared to the real notch case. This result is expected from our discussions on future improvement of these tension-compression formulations in Sect. 3.3. The failure of these elastic energy decompositions to account for the actual damage value or its gradient approximating the crack normal has been reported by $[27,29]$. In the subsequent discussions we will only consider the case where the initial crack is introduced via a real notch in the geometry.

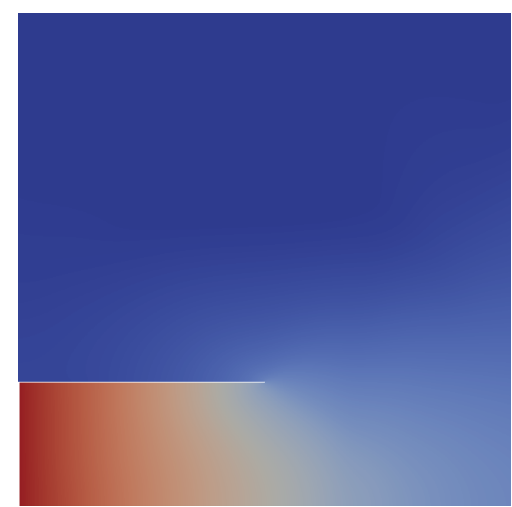

(a) Real notch

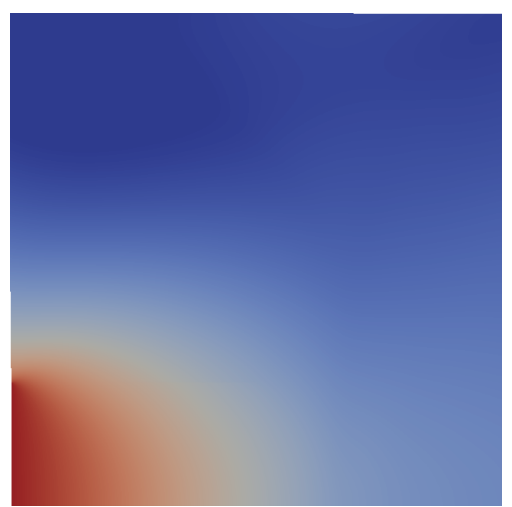

(b) Initial damage

Figure 10. Displacement $u_{x}$ ranging from $0 \mathrm{~mm}$ (blue) to $0.4 \mathrm{~mm}$ (red) at $t \approx 2.4 \times 10^{-5} \mathrm{~s}$ when the real notch case starts to propagate.

The numerically obtained damage profile on a cross-section in the reference configuration parallel to the crack normal is compared to the theoretical one given by $\alpha(x)=(1-|x| / D)^{2}$ with $D=2 \ell$ in the (PAMM) case [11]. From Fig. 11, it can be observed that the numerical damage profile is wider than the analytical prediction by approximately $2 h=0.2 \mathrm{~mm}$. This phenomena leads to the definition of a numerically amplified effective fracture toughness $\left(G_{\mathrm{c}}\right)_{\text {eff }}$, see [6], which in this example is given 
by $\left(G_{\mathrm{c}}\right)_{\mathrm{eff}}=(1+3(2 h) /(8 \ell)) G_{\mathrm{c}}$ corresponding to the constitutive law (PAMM) adapted from [47].

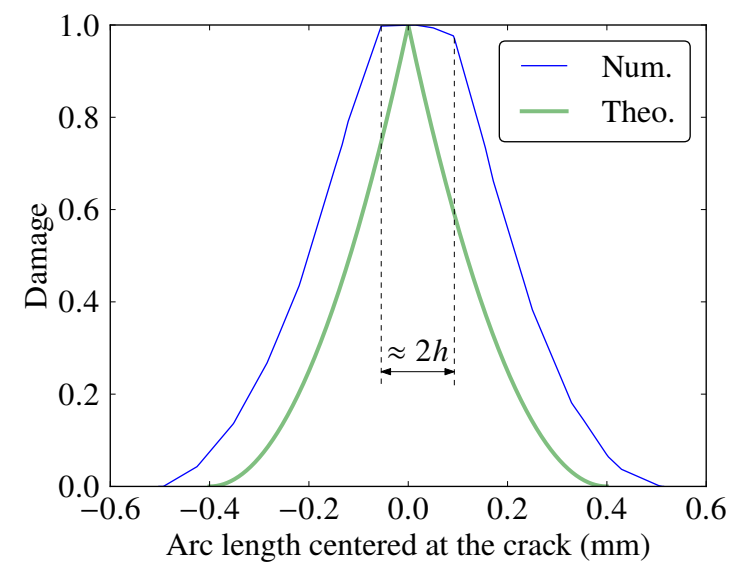

Figure 11. Damage profile perpendicular to the crack.

From the $\Gamma$-convergence result [6] the crack length $l_{t}$ can be estimated by

$$
\mathcal{S}\left(\alpha_{t}\right) \approx\left(G_{\mathrm{c}}\right)_{\mathrm{eff}} \cdot l_{t} .
$$

A 2nd-order difference scheme is then used to calculate the crack velocity. As can be seen from Fig.

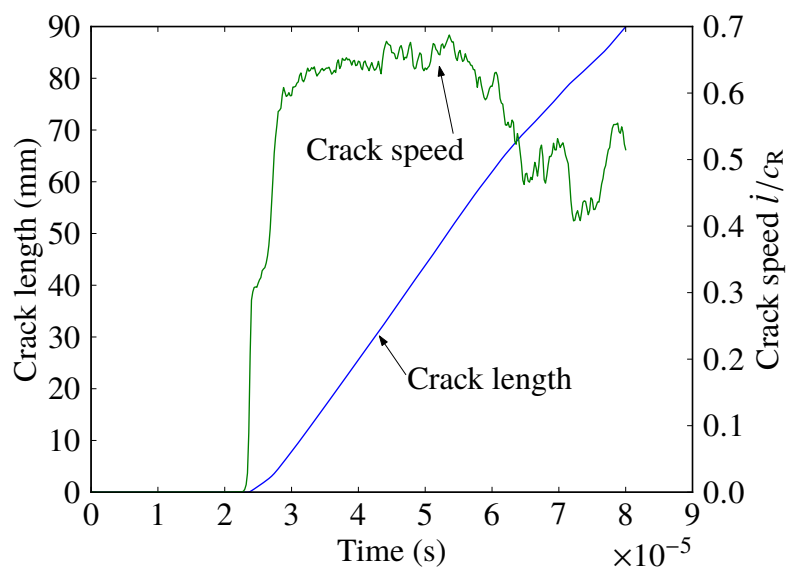

Figure 12. Crack length and velocity obtained for the edge-cracked plate with an imposed impact speed

$$
v=16.5 \mathrm{~m} / \mathrm{s} \text {. }
$$

12 , the crack speed is well bounded by the Rayleigh wave speed (here $0.7 c_{R}$ ), the theoretical limiting speed for an in-plane crack. It should be noted that this upper bound is rooted in the stability condition (16) and the energy balance (17), contrast to the thick level set approach [48] where this limiting speed is considered as an additional modeling parameter. The crack length is approximately $90 \mathrm{~mm}$ at $t=8 \times 10^{-5} \mathrm{~s}$ when the crack is about to reach the boundary, cf. Fig. 9. This estimation agrees fairly well with a direct calculation based on a straight crack propagating at $64^{\circ}$, which gives about $83 \mathrm{~mm}$. We believe that the discrepancy on crack length as well as a smaller limiting speed for brittle materials reported in experiments can be attributed to the dynamic instability mechanism reviewed in [49]. As the crack speed approaches a critical speed approximately $0.4 c_{R}$, micro-branches appear along the main crack and hence more energy is dissipated during propagation. In that case (44) is no longer valid and an apparent energy release rate should be adapted to be velocity-dependent. This point is recently under investigation and will be the object of another contribution. With the 
(AT) constitutive law, authors of $[1,43]$ report a systematic overestimation of the damage dissipation energy according to (44). Following our discussion in the previous simulation, we suspect that it is mainly due to the absence of a purely elastic domain and the fact that damage evolves even in the stress-hardening phase. However in the definition of the fracture toughness this phenomena is not taken into account [6].

When the prescribed impact velocity is increased from $v=16.5 \mathrm{~m} / \mathrm{s}$ to $v=100 \mathrm{~m} / \mathrm{s}$, successive crack branching and nucleation of cracks at the lower-right corner due to high tensile stresses are observed as can be seen from Fig. 13. In Fig. 13b the hydrostatic stress $p_{t}=\frac{1}{2} \operatorname{tr} \sigma_{t}$ is presented in the deformed configuration and we verify that no damage is produced in the compression zones. To visualize the crack, elements with $\alpha_{t}>0.9$ are hidden in the graphical output. We note a finite displacement/rotation of the cracked plate, which justifies our initial motivation to propose a large displacement extension of the gradient damage model in explicit dynamics. Similar phenomena have been reported in [2] with $v=50 \mathrm{~m} / \mathrm{s}$. Recall that in the Kalthoff-Winkler experiment a failure-mode transition from mode-I to mode-II is observed when the impact velocity increases. The discrepancy between our simulation and the experiment is due to the material constitutive behavior. As a material parameter, the tension-compression formulation [10] coupled with a purely elastic model favors propagation of mode-I cracks in the direction perpendicular to the maximal principle stress. On the contrary, the high strength steel used in the experiment develops a considerable plastic zone along the mode-II crack and an elastic-plastic-damage model should be more suitable [5]. Nevertheless, experimentally more bifurcations are indeed observed for brittle materials such as glass when the impact velocity is increased, which is known as a velocity effect in [50].

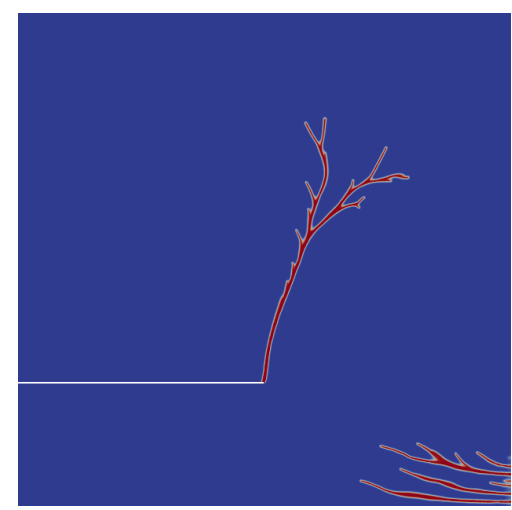

(a) Damage field $\alpha_{t}$ ranging from 0 (blue) to 1 (red).

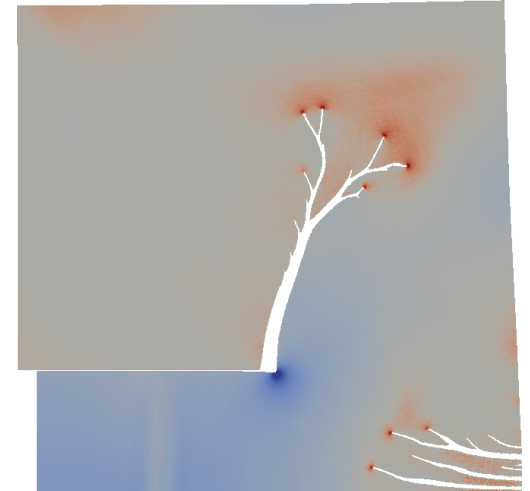

(b) $\frac{1}{2} \operatorname{tr} \sigma_{t}$ ranging from less than $-1 \times 10^{4} \mathrm{MPa}$ (blue) to more than $1.5 \times 10^{3} \mathrm{MPa}($ red).

Figure 13. Simulation results at $t=4 \times 10^{-5} \mathrm{~s}$ with an impact speed $v=100 \mathrm{~m} / \mathrm{s}$. Tension-compression asymmetry model [10] is used.

On the other hand, the widely used elastic energy density split proposed in [26] produces diffusive damage in compression zones. From Fig. 14, we observe appearance of damage at the lower-left corner and at the lower surface of the initial crack edge, even though they are both under compression as can be seen in Fig. 14b. This phenomena is conforming to our previous theoretical analysis of this model on a homogeneous uniaxial compression experiment in Sect. 3.2, where it is found that damage grows even though the compressive stress is still increasing in its absolute value.

The tension-compression split based on the trace of the total strain [25] is also tested. In [9], the pure compression version of this model is used to simulate shear cracking behavior in the stone ashlars. In this dynamic impact problem, we also observe at $t \approx 7 \times 10^{-6} \mathrm{~s}$ appearance of mode-II cracks originating from the impacted-edge, see Fig. 15. We conclude that the tension-compression split (22) could indeed be considered as a material parameter as it represents the fracture mechanism determined by the microstructure. Note however that the calculation suddenly stops after that time due to an extremely small CFL time step $\Delta t_{\mathrm{CFL}}=h / c$, which is caused by a highly distorted element $h \rightarrow 0$ in our updated Lagrangian formulation. According to our Hencky's hyperelastic model (9), the 


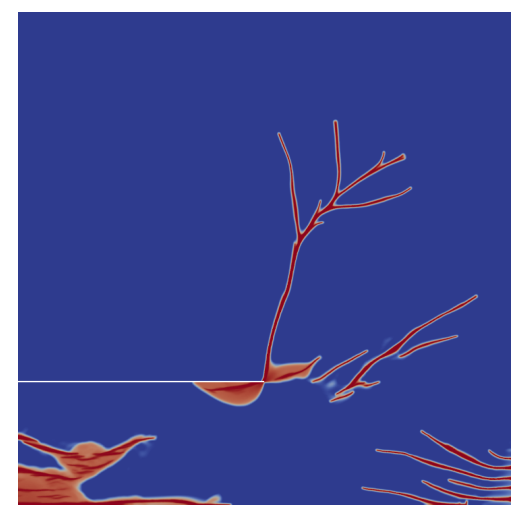

(a) Damage field $\alpha_{t}$ ranging from 0 (blue) to 1 (red).

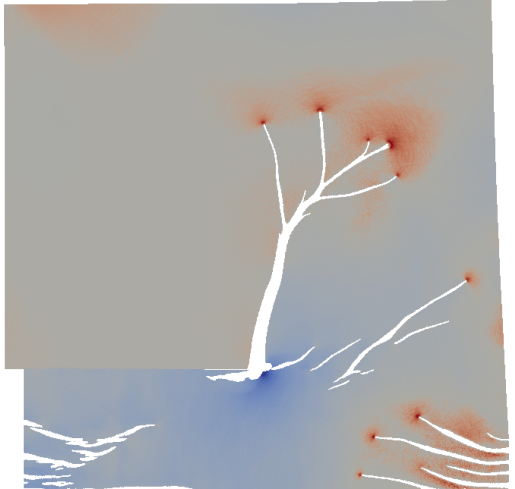

(b) $\frac{1}{2} \operatorname{tr} \sigma_{t}$ ranging from less than $-1 \times 10^{4} \mathrm{MPa}$ (blue) to more than $1.5 \times 10^{3} \mathrm{MPa}$ (red).

Figure 14. Simulation results at $t=4 \times 10^{-5} \mathrm{~s}$ with an impact speed $v=100 \mathrm{~m} / \mathrm{s}$. Tension-compression asymmetry model [26] is used.

Cauchy stress $\sigma_{t}$ goes to infinity as $J_{t} \rightarrow 0$, thus material interpenetration is somehow prevented. However mesh distortion is not since only the product of all principal stretches $J_{t}=\lambda_{1} \lambda_{2} \lambda_{3}$ is controlled. The same numerical issue has been reported by [17] in which an Ogen hyperelastic model is used. Remark that the use of a tension-compression split based on the positive eigenvalues of the strain, i.e. that of $[26,10]$, actually circumvents this problem by revising the material constitutive behavior.

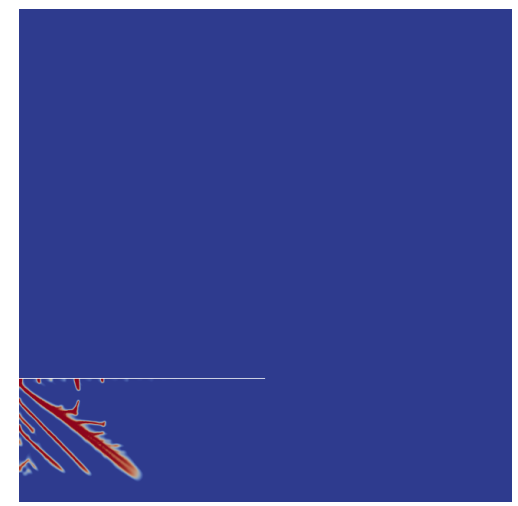

Figure 15. Damage field at $t \approx 7 \times 10^{-6} \mathrm{~s}$ obtained for the edge-cracked plate with an imposed impact speed $v=100 \mathrm{~m} / \mathrm{s}$. The elastic energy split [25] is used.

\subsection{Crack arrest due to the presence of a hole}

Finally we propose to experimentally validate the dynamic gradient damage model following the work of [8]. The problem considered is the "one crack two holes" test studied in [51], where it is found that in dynamics cracks may be pushed away from the holes present in the domain due to wave reflections. The geometry and the boundary conditions are recalled in Fig. 16. Plane stress condition is assumed. Initial crack is introduced via a real notch in the geometry. The damage constitutive law (PAMM) is again used due to its interesting properties discussed in the dynamic crack branching problem. Since PMMA is a brittle material [52] and the model of [26] possesses a peculiar behavior under high compression, the tension-compression asymmetry formulation proposed by [10] is adopted. Materials properties of PMMA, including the density, the dynamic Young's modulus and the Poisson ratio, are borrowed from [51]. In their calculations crack propagation is based on a variant of the 


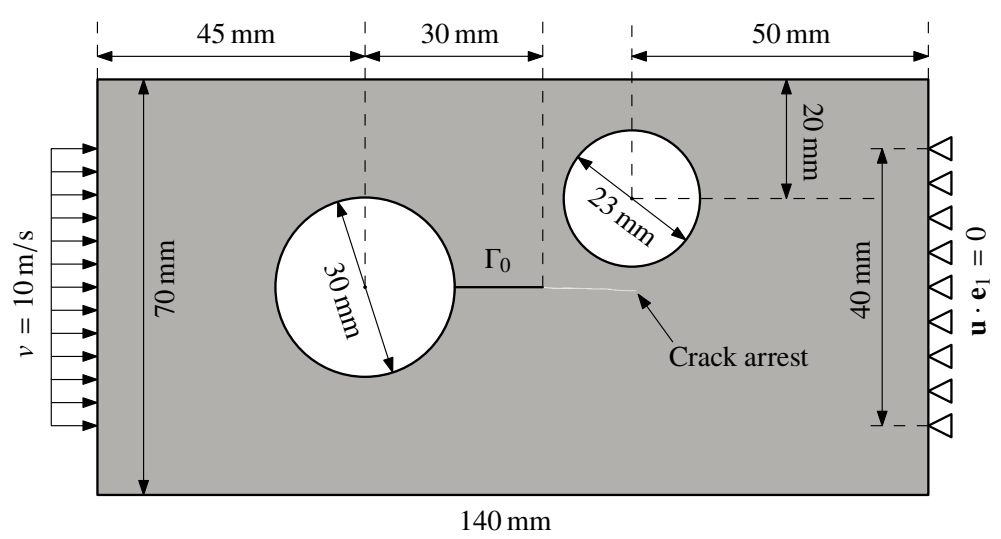

Figure 16. Geometry and boundary conditions for the "one crack two holes" experiment studied in [51]. Damage field $\alpha_{t}$ at $t=2 \times 10^{-4} \mathrm{~s}$ ranging from 0 (gray) to 1 (white).

Griffith's law where one critical stress intensity factor $K_{\mathrm{IC}}=1.03 \mathrm{MPa} \sqrt{\mathrm{m}}$ predicts initiation and another $K_{\mathrm{IA}}=0.8 \mathrm{MPa} \sqrt{\mathrm{m}}$ determines crack propagation and arrest. The latter one is used in our calculation as it deals with the most important phase of crack evolution. It is then converted to the fracture toughness

$$
G_{\mathrm{c}}=\frac{K_{\mathrm{IA}}^{2}}{E} \approx 0.2667 \mathrm{~N} / \mathrm{mm}
$$

thanks to the Irwin's formula under plane stress condition. The material internal length, or equivalently the maximal tensile stress of PMMA used in the experiment through (43), is unknown. Two reasonable values are tested corresponding respectively to a critical stress $70 \mathrm{MPa}$ or $80 \mathrm{MPa}$, which gives along with (45) either $\ell \approx 0.05 \mathrm{~mm}$ or $\ell \approx 0.0375 \mathrm{~mm}$. An unconstrained mixed triangular-quadrilateral mesh refined with $h \approx 2 \times 10^{-2} \mathrm{~mm}$ near the initial crack and all possible nucleation sites is used, arriving at approximately 400000 elements.

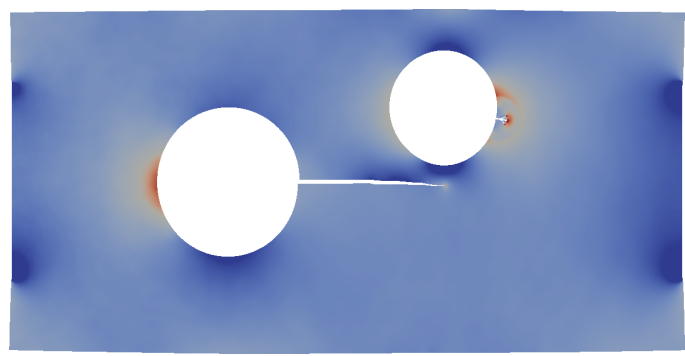

(a) Obtained with $\sigma_{\mathrm{m}}=70 \mathrm{MPa}$ at $t \approx 1.6 \times 10^{-4} \mathrm{~s}$.

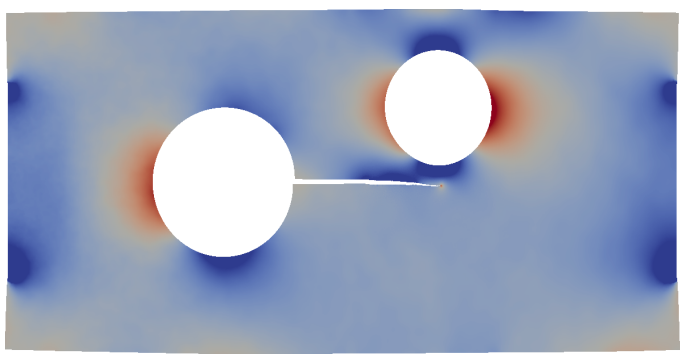

(b) Obtained with $\sigma_{\mathrm{m}}=80 \mathrm{MPa}$ at $t \approx 1.8 \times 10^{-4} \mathrm{~s}$.

Figure 17. Hydrostatic stress $p_{t}=\frac{1}{2} \operatorname{tr} \sigma_{t}$ ranging from less than $-30 \mathrm{MPa}$ (blue) to more than $30 \mathrm{MPa}$ (red) in the crack arrest problem.

The simulations results are illustrated in Fig. 17. In both cases crack arrest is reproduced due to the high compression area under the right circular hole. In the case when the maximal tensile stress is set to $\sigma_{\mathrm{m}}=70 \mathrm{MPa}$, secondary crack nucleation is observed at the right circular hole boundary under high tension, see Fig. 17a. This phenomena is not observed in experiments and hence the critical stress value of $\sigma_{\mathrm{m}}=70 \mathrm{MPa}$ is thus underestimated. In the $\sigma_{\mathrm{m}}=80 \mathrm{MPa}$ case, no secondary crack nucleation is found. This result again highlights the role played by the internal length $\ell$ as a material parameter.

As the crack front is not explicitly tracked in phase-field modeling of fracture, here the current crack tip is located on the contour $\alpha=0.9$ at the farthest point in the $x$-direction. We then compare the numerical crack tip abscissa evolution with the experimental one [51], in Fig. 18. Very good 


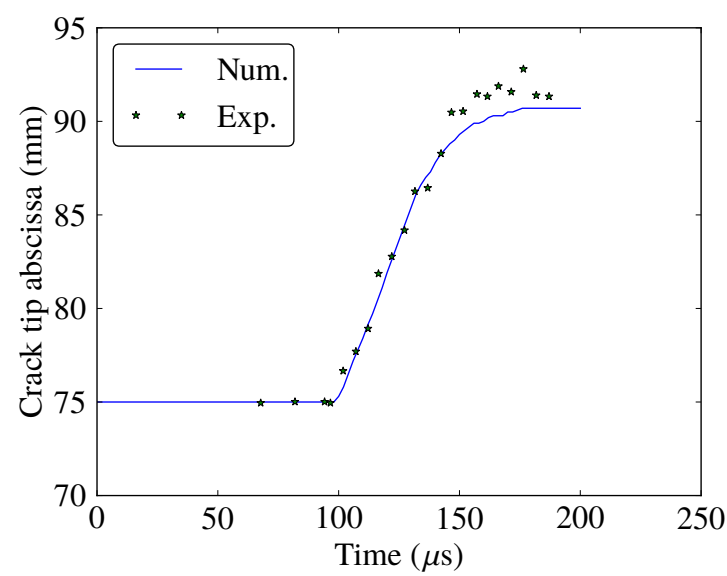

Figure 18. Crack tip abscissa evolution in the crack arrest problem. Comparison between the $\sigma_{\mathrm{m}}=80 \mathrm{MPa}$ case and the experimental results.

agreement can be found in the crack initiation and propagation phase. The crack arrest predicted is slightly conservative compared to the experimental one. This could be due to the small deviation of the initial crack from the symmetry axis in the experiment [51]. Meanwhile the maximal tensile stress $\sigma_{\mathrm{m}} \geq 80 \mathrm{MPa}$ could be considered as an adjusting parameter of the model. More simulations could be performed to determine its best value, at a price of using a more refined mesh since $\ell \propto 1 / \sigma_{\mathrm{m}}^{2}$ according to (43).

\section{CONCLUSION}

In this paper a dynamic gradient damage model formulated at large displacements is proposed. Its use as a phase-field model of dynamic fracture problems is studied. It is computationally more demanding compared to traditional approaches based on a sharp description of cracks. The Griffith's law combined with specialized numerical methods could perform reasonably well with much less computational cost for fracture problems in absence of crack nucleation and complex topology changes. The major advantage of phase-field modeling reside in its generality in treating $2 \mathrm{~d}$ and $3 \mathrm{~d}$ crack evolution problems by providing a unified framework from onset to structural failure. Thanks to an efficient parallelization of the solving algorithm, the computing time can also be significantly reduced as demonstrated in Fig. 5.

Two particular damage constitutive laws (AT) and (PAMM) are compared both from a computational and physical point of view. On one hand, the widely used crack surface density function (AT) is not suitable to model brittle fracture since an elastic domain is absent. On the other hand the actual solving of the damage minimization problem (41) is more costly than the damage constitutive law (PAMM) which possesses an optimal damage profile of finite band. It is also illustrated that the cost of a general quadratic bound-constrained minimization solver is acceptable.

Different tension-compression asymmetry formulations in Sect. 3 are also tested. Some physical properties derived through a uniaxial traction experiment are verified in actual dynamic fracture problems. The elastic energy split proposed by [10] is recommended for brittle materials because homogeneous (diffusive) damage does not occur under compression. However these models should be modified to correctly account for the unilateral contact condition. A better strategy may be to use a transition algorithm between the phase-field and the sharp-interface description of cracks.

We conclude that the gradient damage model as well as its current implementation could indeed be used to approximate and investigate real dynamic brittle fracture problems with sufficient computational efficiency. Future work will be devoted to experimental validation of the model in 3-d cases and a better understanding of the first-order stability condition (16) in case of micro and macro-branching phenomena. 


\section{A. DERIVATION OF THE ELASTODYNAMIC EQUATION}

Denoting the variation $\mathbf{v}-\mathbf{u}$ by $\mathbf{w}$ and testing (16) with $\beta=\alpha$, we obtain after an integration by parts in the time domain supposing the displacement solution $\mathbf{u}$ is sufficiently regular in time

$$
\mathcal{A}^{\prime}(\mathbf{u}, \alpha)(\mathbf{w}, 0)=\int_{I} \mathrm{~d} t \int_{\Omega}\left(\tau\left(\boldsymbol{\varepsilon}\left(\mathbf{u}_{t}\right), \alpha_{t}\right) \cdot \boldsymbol{\varepsilon}^{\prime}\left(\mathbf{u}_{t}\right) \mathbf{w}_{t}+\rho \ddot{\mathbf{u}}_{t} \cdot \mathbf{w}_{t}\right)-\overline{\mathcal{W}}_{t}\left(\mathbf{w}_{t}\right)=0
$$

where the Kirchhoff stress tensor $\tau$ is given by (9) and $\boldsymbol{\varepsilon}^{\prime}\left(\mathbf{u}_{t}\right) \mathbf{w}_{t}$ denotes the derivative of the Hencky strain in the direction of $\mathbf{w}_{t}$. The equality $\mathcal{A}^{\prime}(\mathbf{u}, \alpha)(\mathbf{w}, 0)=0$ follows given that $C_{t}$ is a vector space. We will now use the work conjugacy condition satisfied by the Hencky's hyperelastic model [19]

$$
\dot{w}_{t}=\tau_{t} \cdot \mathbf{D}_{t}=\tau_{t} \cdot \dot{\mathbf{h}}_{t} \Longrightarrow \tau_{t} \cdot \nabla^{\mathrm{s}} \mathbf{v}_{t}=\tau_{t} \cdot \boldsymbol{\varepsilon}^{\prime}\left(\mathbf{u}_{t}\right) \mathbf{v}_{t}
$$

where $\dot{w}_{t}$ is the rate of work per unit volume in the reference configuration and $\mathbf{D}_{t}$ is the stretching, i.e. the symmetrized part of the velocity gradient $\nabla \mathbf{v}_{t}=\dot{\mathbf{F}}_{t} \mathbf{F}_{t}^{-1}$. Passing to the current configuration, we obtain thus

$$
\mathcal{A}^{\prime}(\mathbf{u}, \alpha)(\mathbf{w}, 0)=\int_{I} \mathrm{~d} t \int_{\Omega_{t}}\left(\sigma\left(\varepsilon\left(\mathbf{u}_{t}\right), \alpha_{t}\right) \cdot \nabla^{\mathrm{s}} \mathbf{w}_{t}+\rho_{t} \ddot{\mathbf{u}}_{t} \cdot \mathbf{w}_{t}\right)-\mathcal{W}_{t}\left(\mathbf{w}_{t}\right)
$$

with $\rho_{t}=\rho / J_{t}$ the density in the current configuration and

$$
\mathcal{W}_{t}\left(\mathbf{w}_{t}\right)=\int_{\Omega_{t}} \mathbf{f}_{t} \cdot \mathbf{w}_{t}+\int_{\boldsymbol{\phi}_{t}\left(\partial \Omega_{F}\right)} \mathbf{F}_{t} \cdot \mathbf{w}_{t}
$$

the external power corresponding to the body forces $\mathbf{f}_{t}$ and the surface tractions $\mathbf{F}_{t}$ densities transformed to the deformed configuration [53]. If we suppose further that the Cauchy stress $\sigma_{t}=\sigma\left(\varepsilon\left(\mathbf{u}_{t}\right), \alpha_{t}\right)$ is sufficiently regular in space, an integration by parts in space along with the fundamental lemma of calculus of variations gives finally the desired elastodynamic equation (19).

References

1. Borden MJ, Verhoosel CV, Scott MA, Hughes TJ, Landis CM. A phase-field description of dynamic brittle fracture. Computer Methods in Applied Mechanics and Engineering 2012; 217-220(0):77-95, doi:http://dx.doi.org/10.1016/ j.cma.2012.01.008. URL http://www. sciencedirect.com/science/article/pii/S0045782512000199.

2. Hofacker M, Miehe C. Continuum phase field modeling of dynamic fracture: variational principles and staggered FE implementation. International Journal of Fracture 2012; 178(1-2):113-129.

3. Schlüter A, Willenbücher A, Kuhn C, Müller R. Phase field approximation of dynamic brittle fracture. Computational Mechanics 2014; 54(5):1141-1161, doi:10.1007/s00466-014-1045-x. URL http://dx.doi.org/10.1007/ s00466-014-1045-x.

4. Miehe C, Schänzel LM, Ulmer H. Phase field modeling of fracture in multi-physics problems. Part I. Balance of crack surface and failure criteria for brittle crack propagation in thermo-elastic solids. Computer Methods in Applied Mechanics and Engineering 2015; 294:449-485, doi:http://dx.doi.org/10.1016/j.cma.2014.11.016. URL http://www.sciencedirect.com/science/article/pii/S0045782514004423.

5. Miehe C, Hofacker M, Schänzel LM, Aldakheel F. Phase field modeling of fracture in multi-physics problems. Part II. Coupled brittle-to-ductile failure criteria and crack propagation in thermo-elastic-plastic solids. Computer Methods in Applied Mechanics and Engineering 2015; 294:486-522, doi:http://dx.doi.org/10.1016/j.cma.2014.11.017. URL http://www.sciencedirect.com/science/article/pii/S0045782514004435.

6. Bourdin B, Francfort GA, Marigo JJ. The Variational Approach to Fracture. Journal of Elasticity 2008; 91(1-3):5148.

7. Bouchbinder E, Goldman T, Fineberg J. The dynamics of rapid fracture: instabilities, nonlinearities and length scales. Reports on Progress in Physics 2014; 77(4):046 501.

8. Dally T, Weinberg K. The phase-field approach as a tool for experimental validations in fracture mechanics. Continuum Mechanics and Thermodynamics 2015; :1-10.

9. Lancioni G, Royer-Carfagni G. The Variational Approach to Fracture Mechanics. A Practical Application to the French Panthéon in Paris. Journal of Elasticity 2009; 95(1-2):1-30, doi:10.1007/s10659-009-9189-1. URL http://dx.doi.org/10.1007/s10659-009-9189-1.

10. Freddi F, Royer-Carfagni G. Regularized variational theories of fracture: A unified approach. Journal of the Mechanics and Physics of Solids 2010; 58(8):1154-1174, doi:http://dx.doi.org/10.1016/j.jmps.2010.02.010. URL http://www.sciencedirect.com/science/article/pii/S0022509610000475. 
11. Pham K, Amor H, Marigo JJ, Maurini C. Gradient damage models and their use to approximate brittle fracture. International Journal of Damage Mechanics 2011; 20(4):618-652.

12. Sicsic P, Marigo JJ. From gradient damage laws to Griffith's theory of crack propagation. Journal of Elasticity 2013; 113(1):55-74.

13. Li T, Marigo JJ, Guilbaud D, Potapov S. Variational Approach to Dynamic Brittle Fracture via Gradient Damage Models. Applied Mechanics and Materials 2015; 784:334-341.

14. Pham K, Marigo JJ, Maurini C. The issues of the uniqueness and the stability of the homogeneous response in uniaxial tests with gradient damage models. Journal of the Mechanics and Physics of Solids 2011; 59(6):1163-1190.

15. Pham K, Marigo JJ. From the onset of damage to rupture: construction of responses with damage localization for a general class of gradient damage models. Continuum Mechanics and Thermodynamics 2013; 25(2-4):147-171.

16. Simo JC, Pister KS. Remarks on rate constitutive equations for finite deformation problems: computational implications. Computer Methods in Applied Mechanics and Engineering 1984; 46(2):201-215.

17. Piero GD, Lancioni G, March R. A variational model for fracture mechanics: Numerical experiments. Journal of the Mechanics and Physics of Solids 2007; 55(12):2513-2537, doi:http://dx.doi.org/10.1016/j.jmps.2007.04.011. URL http: //www. sciencedirect.com/science/article/pii/S0022509607000981.

18. Xiao H, Bruhns OT, Meyers A. Logarithmic strain, logarithmic spin and logarithmic rate. Acta Mechanica 1997; 124(1-4):89-105.

19. Xiao H, Chen L. Hencky's elasticity model and linear stress-strain relations in isotropic finite hyperelasticity. Acta Mechanica 2002; 157(1-4):51-60, doi:10.1007/BF01182154. URL http: //dx . doi . org/10 . 1007/BF01182154.

20. Mesgarnejad A, Bourdin B, Khonsari M. Validation simulations for the variational approach to fracture. Computer Methods in Applied Mechanics and Engineering 2015; 290:420-437, doi:http://dx.doi.org/10.1016/j.cma.2014.10. 052. URL http://www. sciencedirect.com/science/article/pii/S004578251400423X.

21. Kuhn C, Schlüter A, Müller R. On degradation functions in phase field fracture models. Computational Materials Science 2015; .

22. Bourdin B, Francfort GA, Marigo JJ. Numerical experiments in revisited brittle fracture. Journal of the Mechanics and Physics of Solids 2000; 48(4):797-826.

23. Lorentz E, Kazymyrenko K. Application of a nonlocal damage law to model concrete fracture. Computational Modelling of Concrete Structures 2014; :209-217.

24. Ambati M, Gerasimov T, de Lorenzis L. A review on phase-field models of brittle fracture and a new fast hybrid formulation. Computational Mechanics 2015; 55(2):383-405.

25. Amor H, Marigo JJ, Maurini C. Regularized formulation of the variational brittle fracture with unilateral contact: Numerical experiments. Journal of the Mechanics and Physics of Solids 2009; 57(8):1209-1229, doi:http://dx.doi.org/10.1016/j.jmps.2009.04.011. URL http://www. sciencedirect.com/science/article/ $\mathrm{pii/S0022509609000659.}$

26. Miehe C, Hofacker M, Welschinger F. A phase field model for rate-independent crack propagation: Robust algorithmic implementation based on operator splits. Computer Methods in Applied Mechanics and Engineering 2010; 199(45-48):2765-2778, doi:http://dx.doi.org/10.1016/j.cma.2010.04.011. URL http://www . sciencedirect.com/science/article/pii/S0045782510001283.

27. May S, Vignollet J, de Borst R. A numerical assessment of phase-field models for brittle and cohesive fracture: $\Gamma$-convergence and stress oscillations. European Journal of Mechanics - A/Solids 2015; 52:72-84, doi:http: //dx.doi.org/10.1016/j.euromechsol.2015.02.002. URL http://www.sciencedirect.com/science/article/ pii/S0997753815000157.

28. Ciarlet PG, Nečas J. Injectivity and self-contact in nonlinear elasticity. Archive for Rational Mechanics and Analysis 1987; 97(3):171-188.

29. Strobl M, Seelig T. On the treatment of crack boundary conditions in phase field models of dynamic fracture. Fourth International Conference on Computational Modeling of Fracture and Failure of Materials and Structures (CFRAC), Cachan, France, 2015.

30. Bourdin B, Larsen CJ, Richardson CL. A time-discrete model for dynamic fracture based on crack regularization. International Journal of Fracture 2011; 168(2):133-143.

31. Larsen CJ, Ortner C, Süli E. Existence of Solutions to a Regularized Model of Dynamic Fracture. Mathematical Models and Methods in Applied Sciences 2010; 20(7):1021-1048.

32. Simone A, Askes H, Peerlings RHJ, Sluys LJ. Interpolation requirements for implicit gradient-enhanced continuum damage models. Communications in Numerical Methods in Engineering 2003; 19(7):563-572.

33. Newmark NM. A method of computation for structural dynamics. Journal of the Engineering Mechanics Division 1959; 85(3):67-94.

34. Balay S, Abhyankar S, Adams MF, Brown J, Brune P, Buschelman K, Dalcin L, Eijkhout V, Gropp WD, Kaushik D, et al.. PETSc Users Manual. Technical Report ANL-95/11 - Revision 3.6, Argonne National Laboratory 2015. URL http://www.mcs.anl.gov/petsc.

35. Moré J, Toraldo G. On the Solution of Large Quadratic Programming Problems with Bound Constraints. SIAM Journal on Optimization 1991; 1(1):93-113.

36. Benson SJ, McInnes LC, Moré JJ. GPCG: A Case Study in the Performance and Scalability of Optimization Algorithms. ACM Transactions on Mathematical Software 2001; 27(3):361-376, doi:10.1145/502800.502805. URL http: //doi .acm.org/10.1145/502800.502805.

37. CEA, EC. EUROPLEXUS: A Computer Program for the Finite Element Simulation of Fluid-Structure Systems under Transient Dynamic Loading. User's Manual. Technical Report, Commissariat à l'énergie atomique and European Commission 2015. URL http: //www-epx. cea.fr.

38. Li T, Maurini C. FEniCS (Dynamic) Gradient Damage. https://bitbucket.org/litianyi/ dynamic-gradient-damage 2015.

39. Logg A, Mardal KA, Wells G. Automated solution of differential equations by the finite element method: The FEniCS book, vol. 84. Springer Science \& Business Media, 2012. 
40. Negri M. The anisotropy introduced by the mesh in the finite element approximation of the Mumford-Shah functional. Numerical Functional Analysis and Optimization 1999; 20(9-10):957-982.

41. Lorentz E, Godard V. Gradient damage models: Toward full-scale computations. Computer Methods in Applied Mechanics and Engineering 2011; 200(21-22):1927-1944, doi:http://dx.doi.org/10.1016/j.cma.2010.06.025. URL http://www. sciencedirect.com/science/article/pii/S0045782510001921.

42. Delmas J, Lefèbvre JP. Notice d'utilisation du cluster de calcul Aster5. Technical Report, EDF R\&D Département Analyses Mécaniques et Acoustique 2014.

43. Vignollet J, May S, de Borst R, Verhoosel CV. Phase-field models for brittle and cohesive fracture. Meccanica 2014; 49(11):2587-2601, doi:10.1007/s11012-013-9862-0. URL http://dx.doi.org/10.1007/ s11012-013-9862-0.

44. Pham K, Marigo JJ. Stability of homogeneous states with gradient damage models: size effects and shape effects in the three-dimensional setting. Journal of Elasticity 2013; 110(1):63-93.

45. Kalthoff JF. Modes of dynamic shear failure in solids. International Journal of Fracture 2000; 101(1-2):1-31, doi:10.1023/A:1007647800529. URL http://dx. doi .org/10.1023/A\%3A1007647800529.

46. Scherzinger W, Dohrmann C. A robust algorithm for finding the eigenvalues and eigenvectors of $3 \times 3$ symmetric matrices. Computer Methods in Applied Mechanics and Engineering 2008; 197(45-48):4007-4015, doi:http://dx.doi.org/10.1016/j.cma.2008.03.031. URL http://www. sciencedirect.com/science/article/ $\mathrm{pii} / \mathrm{S} 0045782508001436$.

47. Hossain M, Hsueh CJ, Bourdin B, Bhattacharya K. Effective toughness of heterogeneous media. Journal of the Mechanics and Physics of Solids 2014; 71:15-32, doi:http://dx.doi.org/10.1016/j.jmps.2014.06.002. URL http://www.sciencedirect.com/science/article/pii/S0022509614001215.

48. Moreau K, Moës N, Picart D, Stainier L. Explicit dynamics with a non-local damage model using the thick level set approach. International Journal for Numerical Methods in Engineering 2015; 102(3-4):808-838, doi: 10.1002/nme.4824. URL http://dx. doi.org/10.1002/nme. 4824.

49. Fineberg J, Marder M. Instability in dynamic fracture. Physics Reports 1999; 313(1):1-108.

50. Schardin H. Velocity effects in fracture. ICFO, Swampscott-MA (USA) 1959, 2012.

51. Haboussa D, Grégoire D, Elguedj T, Maigre H, Combescure A. X-FEM analysis of the effects of holes or other cracks on dynamic crack propagations. International Journal for Numerical Methods in Engineering 2011; 86(45):618-636.

52. Grégoire D, Maigre H, Réthoré J, Combescure A. Dynamic crack propagation under mixed-mode loading Comparison between experiments and X-FEM simulations. International Journal of Solids and Structures 2007; 44(20):6517-6534, doi:http://dx.doi.org/10.1016/j.ijsolstr.2007.02.044. URL http://wwW.sciencedirect. com/science/article/pii/S0020768307001126.

53. Ciarlet PG. Mathematical elasticity: Three-dimensional elasticity, vol. 1. Elsevier, 1993. 